\title{
Folkbotanical classification: morphological, ecological and utilitarian characterization of plants in the Napf region, Switzerland
}

\author{
Anna Poncet ${ }^{1}$, Christian R Vogl ${ }^{1}$ and Caroline S Weckerle ${ }^{2^{*}}$
}

\begin{abstract}
Background: Discussions surrounding ethnobiological classification have been broad and diverse. One of the recurring questions is whether classification is mainly based on the "inherent structure of biological reality" or on cultural, especially utilitarian needs. So far, studies about ethnobotanical classification have mainly been done in indigenous societies. Comparable data from industrialized countries are scarce. In this paper, folkbotanical classification data from the Napf region in central Switzerland is analysed and cross-culturally compared.
\end{abstract}

Methods: Structured and semi-structured interviews were conducted with 60 adults and children chosen by random sampling. Descriptive statistics, t-tests and cultural domain analysis were used to analyze the data.

Results: Close to 500 folk taxa have been documented during field work. As life-form taxa appeared tree, bush, grass, herb, flower, and mushroom. Intermediate taxa mentioned regularly were sub-categories of the life form tree and bush, i.e. conifer, deciduous tree, fruit tree, stone fruits, pomaceous fruits, and berry bush. The rank of the folk generic was by far the largest with 316 taxa (85.4\% monotypical). The specific rank contained 145 taxa, the varietal 14 taxa. The 475 generic, specific and varietal folk taxa could be assigned to 298 wild growing plant species, which make up $28.13 \%$ of the local flora, and to 213 cultivated plant species, subspecies and cultivars.

Morphology, mainly life-form, fruits, leaves, and flowers, was the most important criterion for classifying plants. Other important criteria were their use (mainly edibility) and habitat (mainly meadow, forest and garden). The three criteria emerged spontaneously out of open questioning.

Conclusion: The classification system of the Napf region is comparable to classification systems of indigenous societies, both in its shallow hierarchical structure and in the amount of recognized taxa.

The classification of plants was mainly guided by morphology, habitat and use. The three aspects seem to be mutually linked for certain plant groups, which results in always the same groups, independent from the different sorting criteria. Sensory perception allows for a broader explanation of the known coincidence of morphology and use groups.

Keywords: Ethnobotany, Ethnobotanical classification, Local plant knowledge, Switzerland

\section{Introduction}

All over the world people classify the surrounding plants and animals in folkbiological systems and appear to do this in similar ways [1]. To distinguish and thereby establish categories, to relate these categories to each other and thus establish classification systems is a deeply rooted human impulse [2]. As plants and animals are the outcome

\footnotetext{
* Correspondence: caroline.weckerle@systbot.uzh.ch

${ }^{2}$ Institute of Systematic Botany, University of Zürich, Zollikerstrasse 107, Zürich CH-8008, Switzerland

Full list of author information is available at the end of the article
}

of an evolutionary process, they accordingly show regularities in morphology and behaviour. Cognitive scientists suggest, that we dispose of an innate ability to classify plants and animals, following roughly the progression of biological evolution. This classification ability helps us to perceive our environment, to memorize information about it, to reason and speculate about it and hence to interact with it e.g. [3-7].

Classifying behaviour and classification systems have been investigated by researchers of many different disciplines such 
as ethnology, anthropology, linguistics, cognitive sciences, zoology and botany. One of the important discussions raised in this context concerns general-purpose versus specialpurpose categorization, i.e. the question whether categories are mainly based on the "inherent structure of biological reality" or on cultural, especially utilitarian needs e.g. $[1,8]$ pp. 2-5. Yet different types of classification, such as morphology-based and use-based systems, are often overlapping and interwoven. It has been pointed out, that a clear separation is sometimes neither possible nor appropriate [2] pp. 7-10, and that different ways of classification may also be due to intracultural knowledge variation and can thus be found within a single community [9].

However, the term "folkbiological classification system" usually designates a hierarchical classification system based on general-purpose categories only. The taxa are defined by the inherent characters of biological species which are directly distinguishable by our senses, first of all morphology, but also smell and taste of plants or the calls of animals. Although this approach neglects important aspects of categorizing plants and animals such as their use or symbolic meaning, it has the advantage that different folkbiological systems can easily be compared cross-culturally. Scientific biological classification, which is based on similar principles like folkbiological classification $[3,1,10]$, is used as reference system for such comparisons. While folk classifications are valid in a restricted area only, scientific classification is consistent and globally applicable.

Most of the studies about folkbotanical classification and nomenclature have been conducted in indigenous societies. Folkbotanical studies in western countries concern either urban populations, whose knowledge about plants is poor
[11-13], specialist knowledge about distinct groups of plants like trees or medicinal plants e.g. [14-16], or classification based on different habitats of plants [17]. When urban people are asked to list plants, they often produce life-form level terms like "tree", "flower" or "grass", whereas people from indigenous societies prefer the genus level to refer to plants [1]. The use of life-form terms indicates pronounced unfamiliarity with plants, which is also described as "devolution of knowledge" [18]. Rural populations tend to have broader plant knowledge [18-21]. However, regarding folkbotanical classification, no comprehensive study conducted in a western rural population is known to the authors.

This study aims to close this gap with an analysis of folkbotanical classification in the Napf region of Switzerland. It is part of an ethnobotanical project exploring linkages between plant diversity and local plant knowledge as a basis for applied projects in the fields of conservation, environmental awareness and education. Based on the gathered names of folktaxa of different ranks and the results of a sorting task, we outline the local folkbotanical classification system, and analyse it cross-culturally. Open questioning allowed us to exceed the general-purpose frame and to identify additional aspects important for categorizing and hence distinguishing and perceiving plants.

\section{Research area}

The Napf region belongs to the northern alpine foothills of Switzerland (Figure 1). It is bounded by a circular valley structure, encompasses around $500 \mathrm{~km}^{2}$ and touches 19 political communities. Elevation ranges between 600 and $1400 \mathrm{~m}$ above sea level. Annual precipitation amounts to $1736 \mathrm{~mm}$ and annual average temperature to $4.6^{\circ} \mathrm{C}$ on the

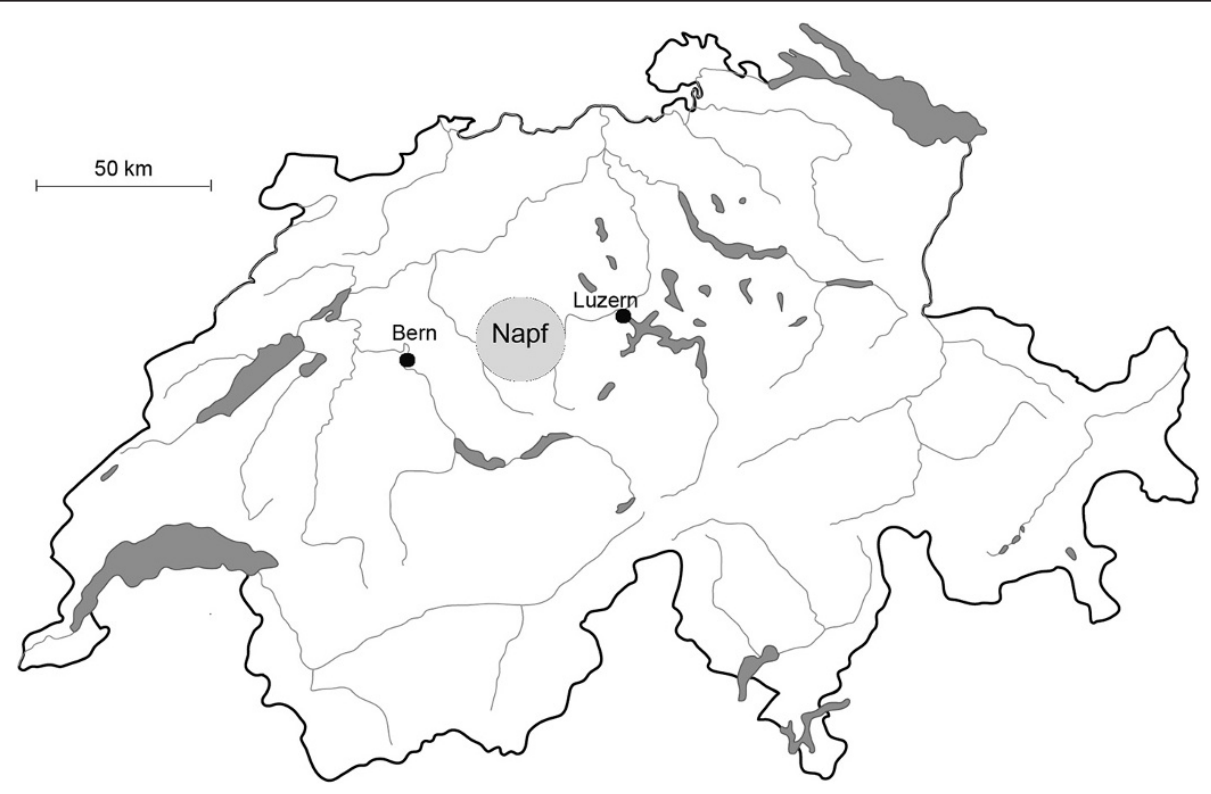

Figure 1 Research area: the Napf region in Switzerland (map by first author). 
centrally located summit of the Napf [22]. The underground, a molasse conglomerate, was topographically shaped by water into radially arranged valleys and ridges. The steepest and the shadowy parts are forested, whereas plainer grounds have been cleared since the $10^{\text {th }}$ century for agriculture. The original form of settlement is still visible: the solitary farms are surrounded by their land and a forest belt, which results in a small-scale mosaic of wood and open space. The forest is dominated by Abies alba Mill., Picea abies (L.) H. Karst. and Fagus sylvatica L.. Meadows and pastures are rather humid and nutrient rich, often of the type Arrhenaterion, Polygono-Trisetion or Cynosurion [23]. Nevertheless, because of its location between the Alps and the plains, the Napf region harbours 1063 different plant species [24,25].

The Napf region exhibits a very rural character also in economic terms. In the nine communes lying entirely within the region, $17-73 \%$ (average $38 \%$ ) of the population works in the agricultural sector, ten times more than in whole Switzerland with 3.6\% [26]. Agriculture in the Napf is focused on dairy farming and upbringing of young livestock. Arable farming, which was important for subsistence until the middle of the $20^{\text {th }}$ century is today practiced only on the lowest and plainest grounds. A farm includes typically 10 to 20 hectares greenland plus some hectares of forest. In most of the farmer's families at least one person has an off-farm employment. Villages are restricted to the bottom of the large valley surrounding the Napf region.

\section{Methods}

Fieldwork was carried out by the first author during August-September 2008 and October-November 2009. A total of 60 informants living on 14 farms were interviewed. The farms were chosen by random sampling and every person living on the farm was asked for an individual interview. The interview partners comprised 33 men and boys, $8-71$ years old (average $38, \pm 20.3$ ), and 27 women and girls, $10-72$ years old (average 36, \pm 19.9 ). All interviews were conducted in Swiss German, were recorded and are deposited at the first authors' home. Prior to the interviews, the interview partners were informed about the project and asked for permission to record the interviews and to take pictures. After each period of fieldwork, they got a summary of the current results and the pictures taken at their homes.

The first series of interviews consisted of a freelist, followed by a semi-structured interview [27,28]. The interviewee was asked to list all indigenous plants he or she could think of ("Säg mer aui iiheimische Pflanze, wo der $i$ Sinn chöme") and was then asked for possible uses of the listed plants.

In order to get higher order categories, a pile sorting task was subsequently performed with the first 36 of the 60 freelist informants [29]. Since it is important, that the interviewees know all the plants which they have to sort, the 43 most frequently listed plants were used and their names printed on small cards. We did not use plant pictures, as they may produce groupings based on superficial morphological similarity [16]. With the question "Which plants belong together?" ("Weli Pflanze ghöre $z$ äme?") the informants were asked individually to sort the 43 cards in as many piles as they wanted to. Each card could be used only once. They were then encouraged to explain their sorting. The 36 pile sortings were done by 20 men and 16 women, aged from 11 to 72 years (average $45, \pm 19.1$ ).

Species identification was done by means of transect walks and participatory observation [30]. Voucher specimens were taken in presence of the informants, identified according to the Flora Helvetica [31] and deposited at the herbarium of the Natural Museum of Lucerne (NMLU). Cultivated plant species were identified at the spot and not vouchered. If they do not figure in the Flora Helvetica, the nomenclature follows the publications of the Swiss edition-lmz [32-34].

The folkbiological terminology used to analyse the data follows Berlin [1]. According to his general principles, folkbiological systems are organized in a hierarchical structure of 6 taxonomic ranks: kingdom, life-form, intermediate, generic, specific, varietal. He defines the ranks as follows: The highest rank of the kingdom contains the two categories plant and animal, although they are not always explicitly named. Life-form taxa form rather large groupings of perceptually similar folk genera, usually based on a small number of biological characters. The intermediate rank is situated between generic and life-form rank. Intermediate taxa assemble up to a dozen of similar folk generics. The generic rank appears to be the largest and most important rank. Folk generics are "the smallest fundamental biological discontinuities easily recognized in any particular habitat" [1] p. 53. They can be recognized without close study and often bear a short, single name. About $80 \%$ of them are monotypic (no subclasses). Polytypic generic taxa are split in two or more specific taxa. The recognition of a specific taxon requires closer examination. It differs in a few characters from other specific taxa of the same generic taxon and bears typically a complex, descriptive name. Folk varietal taxa occur seldom and mainly among plant species that are intensively manipulated, e.g. crop plants of farming societies. Regarding the terminology, it is important not to confuse folk taxa with scientific taxa. A folk generic, for example, corresponds usually with both, the scientific rank of genus and species, sometimes also with taxa of other scientific ranks. Scientific and folk systems are coherent only within themselves. Therefore, throughout this article the term "plants" is used instead of "species" if the concerned folk taxa corresponds with more than one systematic rank. 
For outlining a comparable folkbotanical classification system we assigned the gathered plant names from freelists and pile sortings to the most appropriate rank of Berlin's folk classification by applying his rank characteristics. Important for the definition of generic and specific taxa were first of all the names of the plants, but also the criteria of "easy recognition" versus "need for closer examination" (see above), as well as the order of the plant names in the freelists. If different names were used for a plant, the most frequently mentioned and most concise name was used for the analysis. For example, the two cabbage varieties Brassica oleracea L. convar. capitata var. sabauda L. and B. oleracea L. var. capitata L. were occasionally both called "Chool". However, as most people use "Chool" only for the former and call the latter "Chabis", the two varieties appear in our list as two different folk generics. Intermediate terms were gained from pile sorting, whereby general-purpose groupings based on morphological traits were considered only. Important classification criteria other than morphology are presented in the second part of the results.

The data was organized with Access 2007, t-tests were performed with SPSS 16.0 and Cultural Domain Analysis (Cultural Consensus and Hierarchical Clusteri.ng) was done with Anthropac [35,27].

\section{Results}

Numbers and levels of folk taxa and folk taxonomic overview

The 60 freelists contained 7 to 108 items (mean 44.6, \pm 26.5 ). In total 458 different folk taxa were listed, including 14 fungi. The most frequently listed species was Taraxacum officinale aggr., followed by Rumex obtusifolius L. and Rubus fruticosus aggr. (Table 1).

The semi-structured interviews, the transect walks, the participatory observation and the pile sorting task yielded roughly 40 additional names of taxa, also morphologically defined higher order taxa, which were important for the outlining of the folk taxonomy. Of the 475 generic, specific and varietal folk taxa (listed in Table 2) 222 are wild growing, 191 are cultivated on fields or in homegardens and 62 are wild growing but also cultivated. The wild growing folk taxa could be assigned to 298 plant species, which make up $28.13 \%$ of the local flora with 1063 species. The cultivated folk taxa were assigned to 213 cultivated plants (species, subspecies and cultivars).

Taxonomy (for names and rank assignment see Tables 2 and 3): The plant kingdom was given by the freelist question. The informants listed all kinds of plants including ferns and mosses, in six cases also fungi. As life-form taxa appeared tree (Boum), bush (Stude, Struuch), grass (Gras, Greser), herb (Chrüttli, Chrütter), flower (Blüemli), mushroom (Piuz, Pilz). The intermediate taxa were difficult to grasp, because groupings of generic taxa which are not life-forms are often motivated by other than morphological criteria (see below). However, intermediate general purpose taxa which appeared regularly during the pilesorting task were sub-categories of the life form tree (conifer, deciduous tree, fruit tree, stone fruits, pomaceous fruits) and bush (berry bush). The rank of the folk generic is by far the largest: it contains 316 taxa. Of these taxa $85.4 \%$ are monotypical. In total 186 generic taxa correspond to genera in scientific classification (many of them also monotypical in the region), 110 taxa correspond to lower scientific ranks such as species or even subspecies (e.g. blueberry, Vaccinium myrtillus L. or beetroot, a cultivar of Beta vulgaris L. ssp. vulgaris) and 20 taxa correspond to higher scientific ranks up to classes (e.g. fern, Polypodiidae). The specific rank contains 145 taxa, the varietal 14 taxa. Of the specific taxa 96 correspond to the scientific rank of the species, 30 to lower, 19 to higher ranks. In 11 cases, the difference between two specific taxa refers to their cultivation status, e.g. garden strawberry and wild strawberry.

Children and adolescents up to 20 years old $(n=20)$ listed significantly less plants (mean 24.95, \pm 18.72 ) than interviewees above $20(n=40$, mean 54.35, \pm 24.29 ; t-test $\mathrm{p}<0.001$ ). Their lists also contained proportionally more generic taxa (mean $85.79 \%, \pm 9.41$ ) than the lists of the adults (mean $72.8 \%, \pm 12$; t-test $\mathrm{p}<0.001$ ). No difference in the listed number of plants could be detected between younger adults (21-40 years old, $n=13)$, middle-aged adults (41-60 years old, $\mathrm{n}=18$ ) and older adults (above 60 years, $n=9)$.

\section{Criteria used by the interviewees for pile sorting}

The interview partners split the cards into 4 to 16 (mean $9.2, \pm 2.67$ ), in total 405 groups. The used criteria concerned almost exclusively morphology, use and habitat. One group could get more than one attribute, like, for example, the frequently formed group of the berries which was defined by both morphology and use. The two criteria of this group appear in the statement of an informant, which explained, that they are "small, round and edible things". Morphology influenced the forming of groups in 228 cases, use in 218 cases and habitat in 107 cases. In 41 cases other criteria were used like the seasons (e.g. spring flowers), the importance for insects, esthetical reasons (nice or not nice to look at, beauty), similarities of plant names or no explanation.

During the pile sorting most informants used two or more criteria. Only four of the 36 interview partners sorted the plants consistently by one single criterion: twice by habitat, once by use and once by morphology.

Morphological criteria were: the size and form of the plant, if the plant climbs or not, if the plant is woody or not, properties of the wood, form and properties of the leaves (e.g. prickly, evergreen), form, size and colour of the flowers, form and size of the fruits and seeds. The most 
Table 1 The $\mathbf{4 3}$ most frequently listed plants, subsequently used for the pile sorting

\begin{tabular}{|c|c|c|c|}
\hline German name & Scientific name & Family & Times mentioned $(n=60)$ \\
\hline Löwenzahn & Taraxacum officinale aggr. & Asteraceae & 52 \\
\hline Blacke & Rumex obtusifolius L. & Polygonaceae & 43 \\
\hline Brombeere & Rubus fruticosus aggr. & Rosaceae & 36 \\
\hline Weisstanne & Abies alba Mill. & Pinaceae & 34 \\
\hline Rottanne & Picea abies (L.) H. Karst. & Pinaceae & 33 \\
\hline Himbeere & Rubus idaeus L. & Rosaceae & 33 \\
\hline Apfelbaum Distel & Malus domestica Borkh. & Rosaceae & 33 \\
\hline Schwarzer Holunder & Sambucus nigra L. & Adoxaceae & 33 \\
\hline Brennessel & Urtica dioica L. & Urticaceae & 33 \\
\hline Ahorn & Acer pseudoplatanus L. & Sapindaceae & 32 \\
\hline Rotklee & Trifolium pratense L. s.I. & Fabaceae & 32 \\
\hline Weissklee & Trifolium repens $\mathrm{L}$. & Fabaceae & 32 \\
\hline Spitzwegerich & Plantago lanceolata L. & Plantaginaceae & 31 \\
\hline Buche Linde & Fagus sylvatica $\mathrm{L}$. & Fagaceae & 29 \\
\hline Hahnenfuss & Ranunculus spp. & Ranunculaceae & 29 \\
\hline Birnbaum Margrite & Pyrus communis L. & Rosaceae & 29 \\
\hline Linde & Tilia platyphyllos Scop., T. cordata Mill. & Malvaceae & 28 \\
\hline Margrite & Leucanthemum vulgare Lam. & Asteraceae & 28 \\
\hline Hasel & Corylus avellana L. & Betulaceae & 26 \\
\hline Schlüsselblume & Primula elatior L., P.veris L. & Primulaceae & 26 \\
\hline Heubeere & Vaccinium myrtillus L. & Ericaceae & 25 \\
\hline Gänseblümchen & Bellis perennis $\mathrm{L}$. & Asteraceae & 25 \\
\hline Vogelbeere & Sorbus aucuparia L. & Rosaceae & 23 \\
\hline Eiche & Quercus robur L. & Fagaceae & 23 \\
\hline Zwetschgenbaum & Prunus domestica L. & Rosaceae & 23 \\
\hline Sonnenblume & Helianthus annuus L. & Asteraceae & 22 \\
\hline Distel & Cirsium spp., Sonchus spp. & Asteraceae & 21 \\
\hline Farn & Polypodiidae & e.g. Aspleniaceae, Woodsiaceae & 21 \\
\hline Wilde Erdbeere & Fragaria vesca $\mathrm{L}$. & Rosaceae & 21 \\
\hline Sauerampfer & Rumex acetosa L. & Polygonaceae & 20 \\
\hline Breitwegerich & Plantago major L. s.I. & Plantaginaceae & 20 \\
\hline Kirschbaum & Prunus avium L. & Rosaceae & 20 \\
\hline Stechpalme & Ilex aquifolium L. & Aquifoliaceae & 20 \\
\hline Esche & Fraxinus excelsior $\mathrm{L}$. & Oleaceae & 19 \\
\hline Meertrübeli & Ribes rubrum L. & Grossulariaceae & 18 \\
\hline Hagebutte & Rosa spp. (e.g. R. canina L., R. pendulina L. ) & Rosaceae & 18 \\
\hline Birke & Betula pendula Roth & Betulaceae & 18 \\
\hline Frauenmänteli & Alchemilla spp. & Rosaceae & 16 \\
\hline Efeu & Hedera helix L. & Araliaceae & 16 \\
\hline Knaulgras & Dactylis glomerata $\mathrm{L}$. & Poaceae & 16 \\
\hline Englisch Raygras & Lolium perenne L. & Poaceae & 16 \\
\hline Salbei & Salvia officinalis $\mathrm{L}$. & Lamiaceae & 16 \\
\hline Pfefferminze & Mentha $x$ piperita $\mathrm{L}$. & Lamiaceae & 15 \\
\hline
\end{tabular}


Table 2 Generic, specific and varietal taxa

\begin{tabular}{|c|c|c|}
\hline \multicolumn{2}{|l|}{ Generic taxa } & \multirow{2}{*}{$\begin{array}{l}\text { Herbarium } \\
\text { Number* }\end{array}$} \\
\hline Scientific_name & Local_name & \\
\hline Abies spp./Picea spp. & Tanne, Tanneböim & \\
\hline Acer spp. & Ahorn, Ahorne & \\
\hline Achillea millefolium agg. & $\begin{array}{l}\text { Schafgarbe, } \\
\text { Schofgarbe }\end{array}$ & $\begin{array}{lllll}090708 & 2-1 & 090708 & 2-2 \\
090730 & 9-7 & 090730 & 9-8 \\
100801 & 1-1 & 100801 & 1-2\end{array}$ \\
\hline Aegopodium podagraria L. & $\begin{array}{l}\text { Boumtropfe, } \\
\text { Baumtropfe }\end{array}$ & \\
\hline Aesculus/Castanea & $\begin{array}{l}\text { Kaschtanie, } \\
\text { Kaschtanieboum, } \\
\text { Cheschtene, } \\
\text { Cheschtenebuum, } \\
\text { Cheschtele, } \\
\text { Cheschteleboum, } \\
\text { Chegele, } \\
\text { Chegeleboum }\end{array}$ & \\
\hline Agaricus campestris L. ex FR. & $\begin{array}{l}\text { Fäudschampinjoo, } \\
\text { Schampinjoo, } \\
\text { Schampinjoopüuz, }\end{array}$ & \\
\hline Ajuga reptans $\mathrm{L}$. & Kriechender Günsel & \\
\hline $\begin{array}{l}\text { Alchemilla alpina agg } / A \text {. } \\
\text { conjuncta agg. }\end{array}$ & $\begin{array}{l}\text { Silbermänteli, } \\
\text { Süubermänteli, } \\
\text { Siubermänteli }\end{array}$ & \\
\hline $\begin{array}{l}\text { Alchemilla spp. (e.g. A. } \\
\text { vulgaris L. agg./A. glabra } \\
\text { agg./A. hybrida agg.) }\end{array}$ & $\begin{array}{l}\text { Frouemänteli, } \\
\text { Frauemänteli, } \\
\text { Frouemantu, } \\
\text { Frauenmantel, } \\
\text { Toumänteli }\end{array}$ & $\begin{array}{l}0908151-30908151-4 \\
1008011-8100801 \quad 1-9\end{array}$ \\
\hline Algae (eukaryot.) & Auge & \\
\hline Allium cepa $\mathrm{L}$. & $\begin{array}{l}\text { Zibele, Zwibele, } \\
\text { Zwiebel }\end{array}$ & \\
\hline Allium porrum L. & Louch, Lauch & \\
\hline
\end{tabular}

\begin{tabular}{lll}
$\begin{array}{l}\text { Specific taxa } \\
\text { Scientific_name }\end{array}$ & Local_name & $\begin{array}{l}\text { Herbarium } \\
\text { Number* }\end{array}$ \\
\hline $\begin{array}{l}\text { Abies alba MILL. } \\
\text { Abies nordmanniana }\end{array}$ & $\begin{array}{l}\text { Tanne, Wiisstanne } \\
\text { Nordmannstanne, }\end{array}$ & 080930 4-3 \\
(STEVEN) SPACH & Normannstanne & 080930 4-4 \\
Picea abies (L.) H. Karst. & Rottanne, Fichte & \\
Picea pungens ENGELM. & Blautanne & 080930 4-1 \\
Acer platanoides L. & Spitzahorn & 080930 4-2 \\
Acer pseudoplatanus L. & Bärgahorn, Ahorn & 090730 4-3 \\
& & $0907304-4$
\end{tabular}

Aesculus

Rosscheschtene

Rosskaschtanie,

Söicheschtene

Varietal taxa

Scientific_name

Local_name

Number* 
Table 2 Generic, specific and varietal taxa (Continued)

\section{Allium sativum $\mathrm{L}$. \\ Allium schoenoprasum L. \\ Chnobli, Knoblauch \\ Schnittlouch}

Allium sp. (cult. ornamental) Allium

$\begin{array}{ll}\text { Allium ursinum } \mathrm{L} . & \text { Bärlouch, Bärlauch, } \\ & \text { Bärlouchchrut, }\end{array}$

Bärlouchchrut,

Alnus spp.

Erle

Althaea officinalis $L$.

Eibisch

Althaea rosea (L.) CAV.

Malve, Stockrose

Alyssum maritimum (L.)

Alüssum

LAM.

Amanita muscaria (L. ex FR.) Flüügepiuz,

LAMARCK Flöigepiuz,

Flögepiuz,
Fliegepüuz

Amanita phalloides (VAILL. Chnouebletterpiuz

ex FR.) LINK

Anemone hupehensis

LEMOINE

Anemone nemorosa L.

Geisseblüemli,

Geisseglöggli,

Buschwindröschen

Wucherblüemli

Antennaria dioica (L.)

Musenöörli

GAERTN.

Anthyllis vulneraria L. s.l.

Antirrhinum majus L.

Löiemüli

Apiaceae (Anthriscus/ Chirbele, Kerbel,

Chaerophyllum/Heracleum/ Chirbelenarte

Angelica)
Allium schoenoprasum L. Wiude Schnittlech

Allium schoenoprasum L. Schnittlouch,

(cult.) Schnittlauch,

Schnittlech

$\begin{array}{lll}\text { Alnus incana (L.) } & \text { Erle, Roterle, } & 0810042-3 \\ \text { MOENCH } & \text { normali Erle } & 0810042-4 \\ \text { Alnus viridis (CHAIX) DC. } & \text { Erle, Schwarzerle (!) } & 0810043-3 \\ 081004 & 3-4\end{array}$

$\begin{array}{ll}\text { Angelica sylvestris L. } & \begin{array}{l}\text { Chirbele, Chirbele } \\ \text { für Pfiiffe }\end{array} \\ \text { Anthriscus sylvestris (L.) } & \begin{array}{l}\text { Chirbele, } \\ \text { Wisechirbele, } \\ \text { HOFFM. }\end{array} \\ & \text { Wisecherbu, } \\ & \text { Chirbelestängu }\end{array}$


Table 2 Generic, specific and varietal taxa (Continued)

\begin{tabular}{|c|c|c|c|c|}
\hline & & $\begin{array}{l}\text { Chaerophyllum villarsii } \\
\text { W. D. J. KOCH }\end{array}$ & $\begin{array}{l}\text { e Chirbele, } \\
\text { Boumtropfe }\end{array}$ & $\begin{array}{l}0910194-1 \\
0910194-2 \\
0910194-3\end{array}$ \\
\hline & & $\begin{array}{l}\text { Heracleum } \\
\text { mantegazzianum } \\
\text { SOMMIER et LEVIER }\end{array}$ & Chärbu & \\
\hline & & $\begin{array}{l}\text { Heracleum } \\
\text { sphondylium L. s.l. }\end{array}$ & $\begin{array}{l}\text { Bäretaupe, } \\
\text { Bäretatze, } \\
\text { Bäretööpe, wiudi } \\
\text { Chirbele, Schärlech, } \\
\text { Schärlig }\end{array}$ & $\begin{array}{l}0808161-1 \\
0907306-8 \\
0908151-5 \\
0910031-1 \\
1008013-5 \\
1008013-6\end{array}$ \\
\hline & & $\begin{array}{l}\text { Myrrhis odorata (L.) } \\
\text { SCOP. }\end{array}$ & Chörblichrut & \\
\hline & & $\begin{array}{l}\text { Anthriscus sylvestris (L.) } \\
\text { HOFFM. }\end{array}$ & $\begin{array}{l}\text { Chirbele, } \\
\text { Wisechirbele, } \\
\text { Wisecherbu, } \\
\text { Chirbelestängu }\end{array}$ & \\
\hline & & $\begin{array}{l}\text { Chaerophyllum villarsii } \\
\text { W. D. J. KOCH }\end{array}$ & $\begin{array}{l}\text { e Chirbele, } \\
\text { Boumtropfe }\end{array}$ & $\begin{array}{l}0910194-1 \\
0910194-2 \\
0910194-3\end{array}$ \\
\hline & & $\begin{array}{l}\text { Heracleum } \\
\text { mantegazzianum }\end{array}$ & Chärbu & \\
\hline & & SOMMIER et LEVIER & & \\
\hline & & $\begin{array}{l}\text { Heracleum } \\
\text { sphondylium L. s.l. }\end{array}$ & $\begin{array}{l}\text { Bäretaupe, } \\
\text { Bäretatze, } \\
\text { Bäretööpe, wiudi } \\
\text { Chirbele, Schärlech, } \\
\text { Schärlig }\end{array}$ & $\begin{array}{l}0808161-1 \\
0907306-8 \\
0908151-5 \\
0910031-1 \\
1008013-5 \\
1008013-6\end{array}$ \\
\hline & & $\begin{array}{l}\text { Myrrhis odorata (L.) } \\
\text { SCOP. }\end{array}$ & Chörblichrut & \\
\hline $\begin{array}{l}\text { Apium graveolens L. var. } \\
\text { rapaceum (MILL.) GAUD. }\end{array}$ & $\begin{array}{l}\text { Sellerii, Sällerii, } \\
\text { Säuerii }\end{array}$ & & & \\
\hline Aquilegia vulgaris $\mathrm{L}$. & Akelei, Aupe-Akelei & & & \\
\hline Armoracia rusticana & $\begin{array}{l}\text { Meerrättich, } \\
\text { Meerrätech }\end{array}$ & & & \\
\hline Arnica montana $\mathrm{L}$. & Arnika & & & \\
\hline Artemisia absinthium L. & $\begin{array}{l}\text { Wermuet, } \\
\text { Mueterchrut }\end{array}$ & & & \\
\hline Arum maculatum L. & Aaronstab & & & \\
\hline
\end{tabular}


Table 2 Generic, specific and varietal taxa (Continued)

\section{Aruncus dioicus (WALTER) Bockbart, Geissbart}

\section{FERNALD}

$\begin{array}{ll}\text { Aster sp. (cult.) } & \text { Aschter, Aschterli } \\ \text { Atropa belladonna L. } & \text { Touchirschi }\end{array}$

Touchirschi

Avena sativa L. s.l. Haber, Hafer

Begonia sp. Begonie

Bellis perennis L. Gänseblüemli,

090730 6-3 $0907306-4$

Gänseblümchen, $\quad 090815$ 2-1 090815 2-2

Gänsegismeli, $\quad 100801$ 3-3 100801 3-4

Geisseblüemli,

Waseblüemli,

Wasebürschteli,

Margritli

Berberis julianae C. K. Chrüzdorn,

SCHNEID.

Chrüzlitorn

Beta vulgaris L. var. crassa Fueterrüebe, Rüebe

(ALEF.) WITTM

Ruebe

Beta vulgaris L. ssp. vulgaris Rande

var. conditiva ALEF.

Beta vulgaris L. ssp. vulgaris Chrutstile,

var. flavescens DC. Chrutschtiu

Betula pendula ROTH Birke, Birche 090730 3-1 090730 3-2

Boletus spp. Steipiuz, Steipüuz

Borago officinalis L. Borretsch

Brassica napus L. var. Raps

oleifera (MOENCH) DELILE

Brassica oleracea L. convar. Choleräbli, Chouräbli

acephala var. gongylodes L.

Brassica oleracea L. convar. Bluemechou,

botrytis (L.) ALEF. var. Bluemechöli

botrytis $\mathrm{L}$ Bluemchöli

Brassica oleracea L. convar. Broggoli

botrytis (L.) ALEF. var. italica

PLENCK

Brassica oleracea L. convar. Chou, Chööli, Kohl,

capitata var. sabauda L. Wirz

Brassica oleraceavar. Rööselichou

gemmifera DC. Rosechou,

Rööselichööli 
Table 2 Generic, specific and varietal taxa (Continued)

\section{Brassica oleracea L. var. Chabis, Chool}

capitata L.

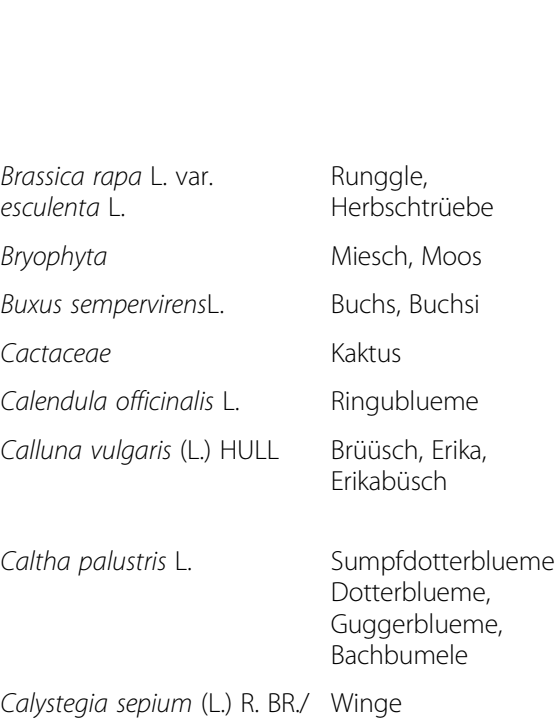

Calystegia sepium (L.) R. BR./ Winge

Convulvulus arvensis $\mathrm{L}$.

Campanula cochleariifolia LAM. C. rhomboidalis L
Gloggeblüemli,
Gloggeblueme
Gloggeblueme

Cannabis sativa $\mathrm{L}$.

Hanf

Cantharellus cibarius FR.

Eierschwumm

Eierschwümm

Eierschwämm

Capsella bursa-pastoris (L.) Hirtetäschli,

Hirtechrut

Cardamine pratensis L.

Carex spp/Juncus spp/

Wiesenschaumkraut Bettseierli, Knöterich

Molinia spp.
Ried, Riedgras,
Sauergräser, Lische,
en Art Schilf

Brassica oleracea $L$

convar. capitata var.

alba L.

$\begin{array}{ll}\text { Brassica oleracea L. } & \text { Rotchool, } \\ \text { convar. capitata var. } & \text { Rotchabis, } \\ \text { rubra L. } & \text { Blauchabis, } \\ & \text { Blauchrut }\end{array}$

100328 1-3 100328 1-4

090815 3-1 090815 3-2 090815 3-3 $1008018-1$ $1008018-2$

$0905102-40905102-5$ 090708 1-1 $0907081-2$

Chabis, Wiisschool

$\begin{array}{ll}\text { Calystegia sepium (L.) R. Winge Wicke } & 090702 \text { 2-3 } \\ 090702 ~ 2-4 \\ \text { BR. } & 1008017-1 \\ & 1008017-2\end{array}$

090815 3-8 090815 3-9

090818 1-1 090818 1-2

090821 1-1 $0908211-2$

Convulvulus arvensis L. Acherwinde

$008017-1$

$1008017-2$ 
Table 2 Generic, specific and varietal taxa (Continued)

Juncus effusus $L / J$.

inflexus L.

Molinia caerulea (L.)

MOENCH/M.

arundinacea SCHRANK

Carex sp. (cult.)

Carlina/Cirsium/Sonchus

Ziergras

Dischtle, Dischtli,

Tischtle

081004 4-1 081004 4-2 Carlina acaulis ssp.

081004 4-3 081005 4-1 caulescens (LAM.)
081005 4-2 090828 1-1 SCHÜBL. et G. MARTENS

\begin{tabular}{|c|c|c|c|}
\hline \multirow[t]{8}{*}{ 1-1 } & \multirow{2}{*}{$\begin{array}{l}\text { SCHÜBL. et G. MARTENS } \\
\text { Cirsium acaule SCOP. }\end{array}$} & \multicolumn{2}{|l|}{$\begin{array}{l}\text { Silberdischtle, chlini } \\
\text { Dischtle, } \\
\text { Edudischtle }\end{array}$} \\
\hline & & $\begin{array}{l}\text { nideri Dischtle, } \\
\text { chlini roti Dischtle }\end{array}$ & $\begin{array}{l}0810053-1 \\
0810053-2\end{array}$ \\
\hline & $\begin{array}{l}\text { Cirsium arvense (L.) } \\
\text { SCOP. }\end{array}$ & $\begin{array}{l}\text { Dischtle wo sech } \\
\text { über Wurzle } \\
\text { verbreite, Dischtle } \\
\text { mit Uslöifer, } \\
\text { Chratzdischtle, } \\
\text { Ackerkratzdistel }\end{array}$ & $\begin{array}{ll}081001 & 1-1 \\
081001 & 1-2 \\
081004 & 1-1 \\
081004 & 1-2 \\
081004 & 1-3 \\
100801 & 9-1 \\
100801 & 9-2\end{array}$ \\
\hline & $\begin{array}{l}\text { Cirsium oleraceum (L.) } \\
\text { SCOP. }\end{array}$ & $\begin{array}{l}\text { Bachdischtle, } \\
\text { Muniseckeli, } \\
\text { Sumpfchrut }\end{array}$ & $\begin{array}{ll}080930 & 1-1 \\
080930 & 1-2 \\
090730 & 2-1 \\
090730 & 2-2 \\
091019 & 1-1 \\
091019 & 1-2\end{array}$ \\
\hline & $\begin{array}{l}\text { Cirsium palustre (L.) } \\
\text { SCOP. }\end{array}$ & $\begin{array}{l}\text { höchi Dischtle, } \\
\text { Stängudischtle, } \\
\text { grossi Dischtle }\end{array}$ & $\begin{array}{l}0810054-1 \\
0810054-2\end{array}$ \\
\hline & $\begin{array}{l}\text { Cirsium vulgare (SAVI) } \\
\text { TEN. }\end{array}$ & $\begin{array}{l}\text { sehr stachligi höchi } \\
\text { Dischtle, importierti } \\
\text { Dischtle }\end{array}$ & $\begin{array}{l}0810041-4 \\
0810041-5 \\
0810054-1\end{array}$ \\
\hline & $\begin{array}{l}\text { Cirsium vulgare (SAVI) } \\
\text { TEN/C. palustre (L.) } \\
\text { SCOP. }\end{array}$ & $\begin{array}{l}\text { normali Dischtle, } \\
\text { angeri Dischtle } \\
\text { (>< Milchdischtle), } \\
\text { höchi Dischtle, } \\
\text { Dischtle wo } \\
\text { Rosette mache u } \\
\text { höch u violett } \\
\text { blüije }\end{array}$ & $\begin{array}{l}0810075-1 \\
0810075-2 \\
0810075-3 \\
0810075-4\end{array}$ \\
\hline & & Esusdischtle & \\
\hline
\end{tabular}

$0908158-3$

$0908212-1$

$0908212-2$

$0908212-3$

$0908212-4$

Ried, Riedströi $\quad 081007$ 3-1

$0810073-2$ 
Table 2 Generic, specific and varietal taxa (Continued)

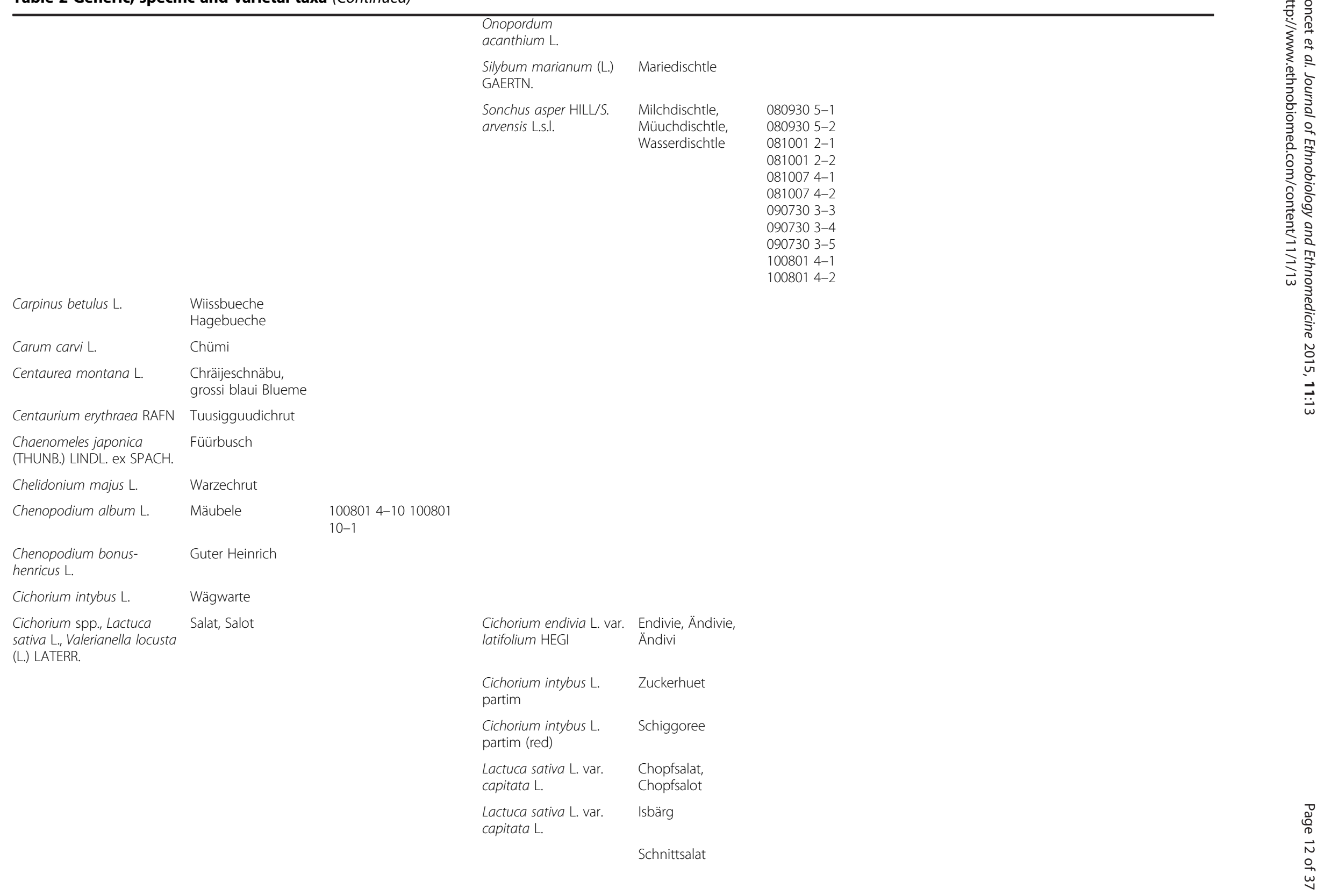


Table 2 Generic, specific and varietal taxa (Continued)

Lactuca sativa subsp.

crispa (L.) SCHÜBL. \& G.

MARTENS

Valerianella locusta (L.) Nüssler, Nüssli,

Clematis sp. (cult.)

Klematis

LATERR. (cult.)

Nüsslisalot

Colchicum autumnale L. Herbschtzitloose

Consolida ajacis (L.) SCHUR Rittersporn

Convallaria majalis $\mathrm{L}$. Meieriisli

Coprinus comatus (O.F. T Tintling

MÜLL. ex FR.) PERS.

Cornus mas L. Tierliboum,

Kornelkirsche

Cornus sanguinea $\mathrm{L}$. Hartriegel

Cornus sp. (cult. orn.) Kornus

Corylus avellana L. plant: Hasle, Haslere,

Haslerete, Hasel,

Haselstude,

Hasustude,

Hasunussstude,

Hasustruuch; fruit:

Hasunuss

Corylus avellana $\mathrm{L}$.

plant: Hasle,

Hasel, Haslerete, $0907304-6$

Hasustude, $1003281-5$

Hasunussstude, $\quad 1003281-7$

Hasustruuch; fruit: $\quad 1008012-1$

Hasunuss

Zierhasu

(cult. ornamental)

$1008012-2$

Crataegus monogyna JACQ. Wiissdorn, Eggedorn 100801 1-10 100801 1-11

Craterellus cornucopioides Totetrumpete

(L. ex FR.) PERS.

Crepis biennis $\mathrm{L}$.

Pippou, Pippau,

Pippaum,

090702 5-7 $0907025-8$

Wisepippou,

090730 8-1 $0907308-2$

Wiesenpippau

Sibegringe, gäubi

090813 1-1

steichigi Blüeml

Crocus sp.

Krokus, Krokussli

Cucumis sativus $\mathrm{L}$.

Gurke

Cucurbita maxima DUCH.

Chürbis

Cucurbita pepo L.

Zuggetti

Cyclamen sp.

Ziklame

Cydonia oblonga MILL. 
Table 2 Generic, specific and varietal taxa (Continued)

\begin{tabular}{|c|c|c|}
\hline & $\begin{array}{l}\text { Chüttene, Quitte, } \\
\text { Quitteboum }\end{array}$ & \\
\hline Cypripedium calceolus L. & Froueschue & \\
\hline Dahlia $x$ hortensis & Daalie & \\
\hline Daphne mezereum L. & Säidelbascht & \\
\hline Daucus carota L. & Wüudi Mööre & 090730 9-9090730 9-10 \\
\hline $\begin{array}{l}\text { Daucus carota L. ssp. } \\
\text { sativus (Hoffm.) ARCANG. }\end{array}$ & Rüebli & \\
\hline Dianthus barbatus L. & $\begin{array}{l}\text { Stiinägeli, Steinägeli, } \\
\text { wüudi Steinägeli, } \\
\text { Nägeli, Neuke }\end{array}$ & \\
\hline $\begin{array}{l}\text { Dicentra spectabilis (L.) } \\
\text { LEMAIRE }\end{array}$ & Frouehärzli & \\
\hline Digitalis spp. & Fingerhuet & \\
\hline Digitaria/Echinochloa & Hirse & 100801 4-11 100801 4-12 \\
\hline Echinacea sp. & $\begin{array}{l}\text { Sunnehuet, } \\
\text { Sonnenhut }\end{array}$ & \\
\hline Epilobium angustifolium L. & Widerööseli & $1008016-31008016-4$ \\
\hline $\begin{array}{l}\text { Equisetum arvense L./E. } \\
\text { sylvaticum } \mathrm{L} \text {. }\end{array}$ & $\begin{array}{l}\text { Bettseiergras, } \\
\text { Chatzeschwanz, } \\
\text { Chatzeschwänz, } \\
\text { Chatzefarn, } \\
\text { Chatzeschtile, } \\
\text { Fuchsschwanz, } \\
\text { Schachtuhaum, } \\
\text { Isechrut, Rainfarn }\end{array}$ & $\begin{array}{l}0809105-10809105-2 \\
0809105-30810043-10 \\
0810043-20810055-3 \\
0810055-40907302-5 \\
0907302-60908158-5 \\
0908158-60908158-7 \\
0908158-81008011-14 \\
1008011-151008017-5 \\
1008017-6\end{array}$ \\
\hline Eriophorum spp. & $\begin{array}{l}\text { Wougras, } \\
\text { Bärgmanndli }\end{array}$ & 090708 4-1 090708 4-2 \\
\hline $\begin{array}{l}\text { Erodium cicutarium (L.) } \\
\text { L'HER. }\end{array}$ & Reierschnabu & \\
\hline $\begin{array}{l}\text { Eruca vesicaria ssp. sativa } \\
\text { (MILL.) THELL. }\end{array}$ & Rucola & \\
\hline Euonymus europaea L. & Pfaffehüetli & \\
\hline Euphorbia sp. & Woufsmiuch & \\
\hline $\begin{array}{l}\text { Euphrasia spp. e.g. E. } \\
\text { rostkoviana HAYNE }\end{array}$ & Ougetroscht & $0908153-4090815$ 3-5 \\
\hline Fagus sylvatica $\mathrm{L}$. & $\begin{array}{l}\text { Bueche, Buche, } \\
\text { Rotbueche }\end{array}$ & $0907304-70907304-8$ \\
\hline
\end{tabular}


Table 2 Generic, specific and varietal taxa (Continued)

\begin{tabular}{lll}
\hline Filipendula ulmaria (L.) & Mädesüess, & 080930 3-3 080930 3-4 \\
MAXIM. & Geisleiterebluescht, & 090815 8-1 090815 8-2
\end{tabular}

MAXIM.

Geisleiterebluescht,

$1008011-31008011-4$

Foeniculum vulgare MILL.

Fänchu, Fenchu

var. azoricum (MILLER)

THELLUNG

Piuz a tote Böim

Forsythia $\mathrm{x}$ intermedia

Forsiizie

ZABEL

Fragaria spp.

Äbberi, Ärbeeri, Ärdbeeri, Ebbeeri, Erdbeere

Fragaria ananassa

Gartenäbbeeri

$\begin{array}{lll}\text { Fragaria vesca } \mathrm{L} . & \text { wiudi, wüudi, wildi } & 0908154-4 \\ & \text { Äbberi, wildi } & 0908154-5 \\ & \text { Ebberi, chliini } & \\ & \text { Äbbeereli, } & \\ & \text { Waudäbbeeri, roti } & \\ & \text { Beeri am Port, } & \\ & \text { Äbbeeribletter, } & \\ & \text { Äbbeerichrut }\end{array}$

Fraxinus excelsior L

Esche, Ösche

090730 4-1 090730 4-2

Äbbeerichrut

Fuchsia sp.

Fuchsie

Galanthus nivalis L. Schneglöggli,

Schneglogge

Galeopsis tetrahit L. Gluure, Luege,

Houzaan

080904 1-6 090702 3-3

090702 3-4 $0907301-$

$110907301-12100801$

2-7 $1008012-8$

Galinsoga ciliata (RAF.) S.F. Franzosechrut

BLAKE

Galium aparine L. plant: Chliibere,

$0907021-10907021-2$

100801 5-3 100801 5-4

wisses Chrütli

081005 7-1 081005 7-2

Galium mollugo L. agg.

Gentiana asclepiadea L. Stänguenzian

Gentiana spp.

Enzian, Änzia

Änziane

Gentiana clusii E. P.

Grosse Änzian

PERRIER et SONGEON/

G. acaulis L. 
Table 2 Generic, specific and varietal taxa (Continued)

Gentiana lutea L.

Gentiana verna $\mathrm{L}$

$0809041-20907082-5$

090708 2-6 $0907307-1$

090730 7-2 $1008011-$

$121008011-13100801$

5-1 100801 5-2

Geum rivale L.

Buebehösli,

Bachnelkenwurz

Bachtschötteli,

Kaputschinerli

Gladiolus communis L.

Gladiole

Glechoma hederacea L. s.I.

Hedera helix L.

Eföi, wi Ahornbletter 090815 5-1 090815 5-2

wo so ufewachse

Helianthus annuus L.

Sunneblueme

Helianthus tuberosus L.

Topinambur

Furzchnoue

Helleborus niger $\mathrm{L}$.

Chrischtrose

Hepatica nobilis SCHREB.

Hippophae rhamnoides L.

Hordeum vulgare L. s.l.

Sanddorn, Sangdorn

Gärschte, Gerste

Hopfe

Humulus lupulus L.

Hiazinte

Hyacinthus orientalis L.

Hydrangea macro
(THUNB.) SER

Hypericum perforatum L./H. Johannis-Chrut maculatum CRANTZ s.l.

Hyssopus officinalis L.

Ilex aquifolium L.
Hortensie

081005 2-1 081005 2-2 090730 9-3 090730 9-4 $1008017-7$

Isop

Paume, Paumestöck, 091019 2-1 100328 1-2 Stächpaume,

Stächpalme,

Muttipaume
Gaubi wo si d

uüze stäche

Chline Änzian, chl

Enzian

Hordeum vulgare L. s.I. Summergärschte (2 rows of seeds)

$\begin{aligned} \text { Hordeum vulgare L. s.l. } & \text { Wintergärschte } \\ & \text { (4-6 rows of }\end{aligned}$ seeds) 
Table 2 Generic, specific and varietal taxa (Continued)

\begin{tabular}{|c|c|c|c|c|c|}
\hline & $\begin{array}{l}\text { (branches with } \\
\text { prickle-less leaves) }\end{array}$ & & & & \\
\hline Imleria badia (FR.) VIZZINI & Maroneröörling & & & & \\
\hline \multirow[t]{2}{*}{ Impatiens spp. } & Springchrut & & $\begin{array}{l}\text { Impatiens glandulifera } \\
\text { ROYLE }\end{array}$ & $\begin{array}{l}\text { Chinesisches } \\
\text { Springchrut, } \\
\text { Himalaya } \\
\text { Chlepfchrut, } \\
\text { Roserots } \\
\text { Springchrut }\end{array}$ & $\begin{array}{l}0809041-1 \\
1008015-5 \\
1008015-6\end{array}$ \\
\hline & & & $\begin{array}{l}\text { Impatiens noli-tangere } \\
\text { L./l. parviflora DC. }\end{array}$ & $\begin{array}{l}\text { Rüerminidaa, Rühr- } \\
\text { mich-nicht-an, } \\
\text { Gäubs Springchrut }\end{array}$ & $\begin{array}{l}0907307-3 \\
0907307-4\end{array}$ \\
\hline Iris sp. (cult. ornamental) & Iris, Schwärtlilie & & & & \\
\hline Juglans regia $\mathrm{L}$. & $\begin{array}{l}\text { Nussboum, } \\
\text { Nussbuum, Grosse } \\
\text { Nussboum, } \\
\text { Boumnuss, } \\
\text { Baumnuss }\end{array}$ & & & & \\
\hline Juniperus communis L. s.l. & $\begin{array}{l}\text { Wachouder, } \\
\text { Wachouderstruuch, } \\
\text { Wacholder, } \\
\text { Räckhoudere }\end{array}$ & $0809304-50809304-6$ & & & \\
\hline Juniperus sabina $\mathrm{L}$. & Sefi & $1003281-91003281-10$ & & & \\
\hline $\begin{array}{l}\text { Knautia arvensis (L.) } \\
\text { COULT./ K. dipsacifolia } \\
\text { KREUTZER }\end{array}$ & $\begin{array}{l}\text { Witweblueme, wüudi } \\
\text { Skabiose, Blaui ir } \\
\text { Ökowise }\end{array}$ & $0910032-1$ & & & \\
\hline $\begin{array}{l}\text { Laburnum anagyroides } \\
\text { MEDIK. }\end{array}$ & Guudräge, Goudräge & & & & \\
\hline $\begin{array}{l}\text { Lamium galeobdolon }(\mathrm{L} .) \mathrm{L} \text {. } \\
\text { s.l./L. maculatum }(\mathrm{L} .) \mathrm{L} . / \mathrm{L} \text {. } \\
\text { purpureum } \mathrm{L} . / \text { Prunella } \\
\text { vulgaris } \mathrm{L} \text {. }\end{array}$ & $\begin{array}{l}\text { Toubnessle, } \\
\text { Ummuchrut }\end{array}$ & & $\begin{array}{l}\text { Lamium maculatum (L.) } \\
\text { L./L. purpureum } \mathrm{L} . / \\
\text { Prunella vulgaris } \mathrm{L} \text {. }\end{array}$ & $\begin{array}{l}\text { Beiichrüttli, } \\
\text { Ummuchrut }\end{array}$ & $\begin{array}{l}0907024-1 \\
0907024-2 \\
0907024-5 \\
0907024-6\end{array}$ \\
\hline Larix decidua MILL. & Lärche, Lerche & & & & \\
\hline Lavandula sp. & Lavändu, Lavendel & & & & \\
\hline Lemna sp. & Wasserlinse & & & & \\
\hline $\begin{array}{l}\text { Leontopodium alpinum } \\
\text { CASS. }\end{array}$ & Eduwiiss & & & & \\
\hline Lepidium sativum L. & Chressi & & & & \\
\hline Leucanthemum vulgare agg. & $\begin{array}{l}\text { Margrite, Margritli, } \\
\text { Zantihansblueme }\end{array}$ & $\begin{array}{l}0907306-10907306-2 \\
0907306-7\end{array}$ & & & \\
\hline
\end{tabular}


Table 2 Generic, specific and varietal taxa (Continued)

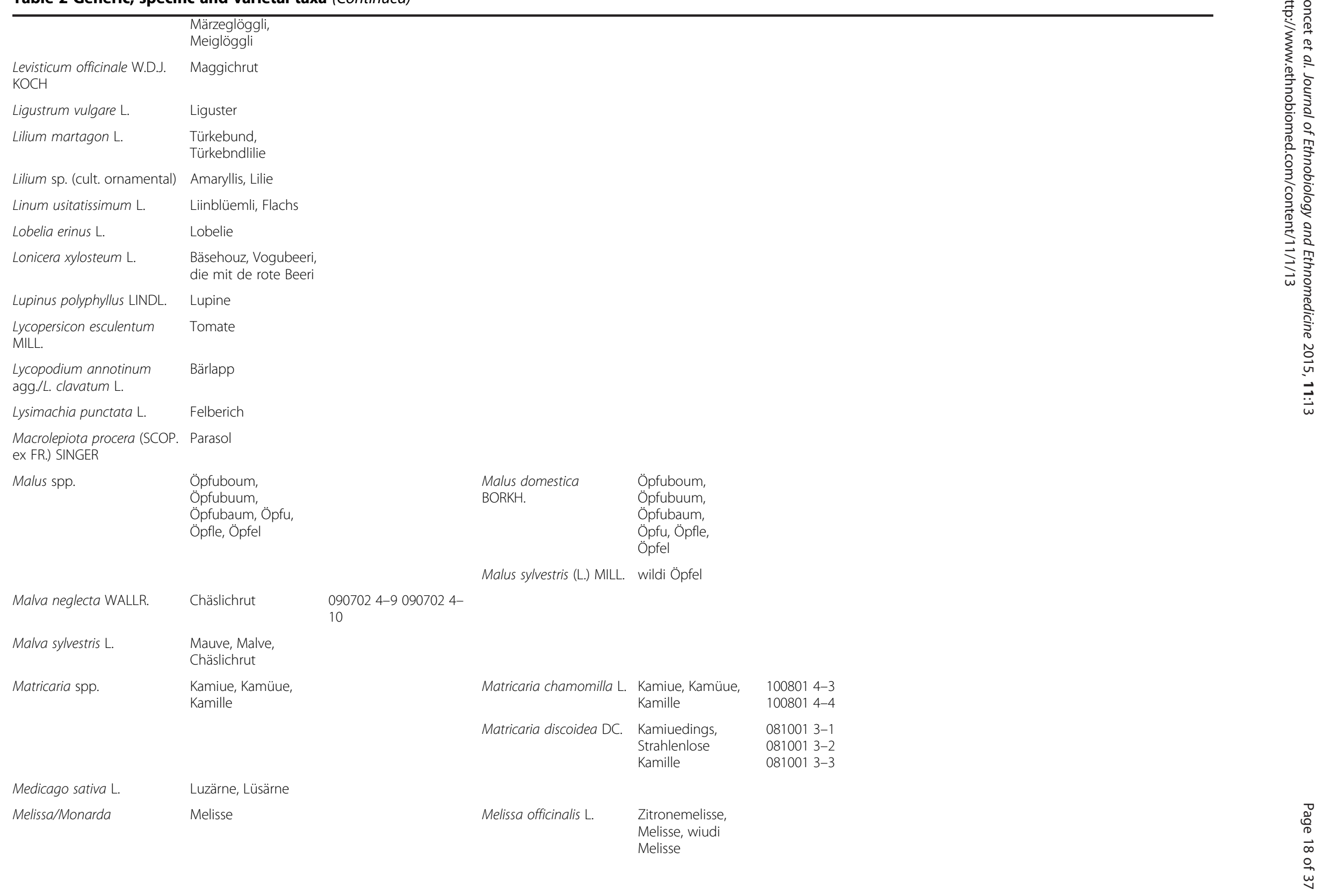


Table 2 Generic, specific and varietal taxa (Continued)

\begin{tabular}{|c|c|c|c|c|}
\hline & & Monarda didyma L. & $\begin{array}{l}\text { Goudmelisse, } \\
\text { Guudmelisse }\end{array}$ & \\
\hline \multirow[t]{8}{*}{ Mentha spp. } & Münze & Mentha aquatica $\mathrm{L}$. & wiudi Münze & \\
\hline & & $\begin{array}{l}\text { Mentha longifolia (L.) } \\
\text { HUDS. }\end{array}$ & $\begin{array}{l}\text { wiudi Münze, } \\
\text { wüudi Münze, } \\
\text { Rossmünze, } \\
\text { Chatzemünze }\end{array}$ & $\begin{array}{l}0810014-1 \\
0810014-2 \\
0907071-3 \\
0907071-4 \\
1008011-6 \\
1008011-7\end{array}$ \\
\hline & & Mentha spicata L. (cult.) & Krauseminze & \\
\hline & & $\begin{array}{l}\text { Mentha spicata L. } \\
\text { "Marokko" }\end{array}$ & $\begin{array}{l}\text { Libanesischi } \\
\text { Pfäffermünze, } \\
\text { Libanesische } \\
\text { Pfäffermünz }\end{array}$ & \\
\hline & & $\begin{array}{l}\text { Mentha suaveolens } \\
\text { EHRH. }\end{array}$ & Öpfumünze & $\begin{array}{l}0910193-1 \\
0910193-2\end{array}$ \\
\hline & & $\begin{array}{l}\text { Mentha suaveolens } \\
\text { EHRH. "Variegata" }\end{array}$ & Ananasmünze & \\
\hline & & Mentha x piperita L. & $\begin{array}{l}\text { Pfäffermünz, } \\
\text { Pfefferminze, } \\
\text { normali Münze }\end{array}$ & \\
\hline & & $\begin{array}{l}\text { Mentha x piperita L. var. } \\
\text { citrata (EHRH.) BRIQ. }\end{array}$ & Orangschemünze & \\
\hline Mespilus germanica L. & Mischple & & & \\
\hline $\begin{array}{l}\text { Montia perfoliata (DONN ex } \\
\text { WILLD.) HOWELL }\end{array}$ & Portulak & & & \\
\hline Morchella sp. & Morchle & & & \\
\hline Muscari sp. & blaui Primeli & & & \\
\hline Myosotis spp. & Vergissmeinnicht & & & \\
\hline Narcissus poeticus L. & Narzisse & & & \\
\hline Narcissus pseudonarcissus L. & $\begin{array}{l}\text { Oschterglogge, } \\
\text { Oschterglöggli, } \\
\text { Apriuglogge, } \\
\text { Narzisse }\end{array}$ & & & \\
\hline Nasturtium officinale R. BR. & Brunnekresse & & & \\
\hline $\begin{array}{l}\text { Nigritella nigra (L.) RCHB. } \\
\text { agg. }\end{array}$ & Männertröi & & & \\
\hline \multirow[t]{2}{*}{ Nymphaea/Nuphar } & Seerose & Nuphar lutea (L.) SM. & Gäubi Seerose & \\
\hline & & Nymphaea sp. & Seerose & \\
\hline
\end{tabular}


Table 2 Generic, specific and varietal taxa (Continued)

\begin{tabular}{|c|c|c|c|c|}
\hline Ocimum basilicum L. & Basilikum & & & \\
\hline Oenothera biennis agg. & Nachtcherze & & & \\
\hline Onobrychis viciifolia SCOP. & Esparsette & & & \\
\hline $\begin{array}{l}\text { Ononis spinosa L. s.l./ O. } \\
\text { repens } \mathrm{L} \text {. }\end{array}$ & Hauhechel & & & \\
\hline \multirow[t]{2}{*}{ Orchidaceae } & Orchideä & & $\begin{array}{l}\text { Dactylorhiza maculata } \\
\text { agg. }\end{array}$ & wüudi Orchidee \\
\hline & & & Orchidaceae (cult.) & Orchidee, Orchideä \\
\hline Origanum majorana L. & Mejoran & & & \\
\hline Origanum vulgare L. & $\begin{array}{l}\text { Doste, Dost, Meiran, } \\
\text { en Art Münze im } \\
\text { Trochene }\end{array}$ & $\begin{array}{l}090707 \text { 1-5 } 090707 \text { 1-6 } \\
0907309-10907309-2\end{array}$ & & \\
\hline Origanum vulgare L. (cult.) & Oregano & & & \\
\hline Paeonia spp. & Pfingschtrose & & & \\
\hline $\begin{array}{l}\text { Papaver sominferum L./P. } \\
\text { dubium L. s.str./ P. rhoeas } L \text {. }\end{array}$ & Moon & $1008014-9$ & & \\
\hline Paris quadrifolia L. & Einbeere & & & \\
\hline Passiflora sp. & Passionsblueme & & & \\
\hline Pelargonium spp. & $\begin{array}{l}\text { Granium, Grani, } \\
\text { Geranie, Granie, } \\
\text { Graninie }\end{array}$ & & & \\
\hline Petasites albus (L.) GAERTN. & $\begin{array}{l}\text { Peschtwurz, } \\
\text { Rebarbere am Wäg }\end{array}$ & & & \\
\hline $\begin{array}{l}\text { Petroselinum crispum (Mill.) } \\
\text { NYM. }\end{array}$ & Peterli, Petersilie & & & \\
\hline $\begin{array}{l}\text { Phallus impudicus L. ex } \\
\text { PERS. }\end{array}$ & Stinkmorchle & & & \\
\hline \multirow[t]{3}{*}{ Phaseolus spp. } & Boone & & Phaseolus coccineus L. & Füürboone \\
\hline & & & $\begin{array}{l}\text { Phaseolus vulgaris L. ssp. } \\
\text { vulgaris var. nanus (L.) } \\
\text { ASCHERS }\end{array}$ & Buschboone \\
\hline & & & $\begin{array}{l}\text { Phaseolus vulgaris L. ssp. } \\
\text { vulgaris var. vulgaris }\end{array}$ & Stangeboone \\
\hline $\begin{array}{l}\text { Phragmites australis (CAV.) } \\
\text { STEUD. }\end{array}$ & Schiuf & & & \\
\hline Physalis alkekengi L. & $\begin{array}{l}\text { Latärneblueme, } \\
\text { Latärme }\end{array}$ & & & \\
\hline Pimpinella saxifraga $\mathrm{L}$. & Bibernelle & & & \\
\hline
\end{tabular}

Phaseolus vulgaris L. SSp. Buschboone

Phaseolus vulgaris L. ssp. Stangeboone

STEUD.

Pimpinella saxifraga $\mathrm{L} . \quad$ Bibernelle 
Table 2 Generic, specific and varietal taxa (Continued)

\begin{tabular}{|c|c|c|c|c|}
\hline$\overline{\text { Pinus cembra L. }}$ & Arve & & & \\
\hline Pinus strobus L. & Weimuet & & & \\
\hline Pinus sylvestris L. & Fööre, Dääle, Dääl & & & \\
\hline Pisum sativum $\mathrm{L}$. & (Ärbs) & Pisum sativum $\mathrm{L}$. & Ärbsli, Ärbs & \\
\hline $\begin{array}{l}\text { Pisum sativum L. ssp. } \\
\text { arvense (L.) ASCH. \& } \\
\text { GRAEBN. }\end{array}$ & Ärbs & & & \\
\hline $\begin{array}{l}\text { Pisum sativum L. convar. } \\
\text { axiphium ALEF. }\end{array}$ & Chifu, Chefe & & & \\
\hline \multirow[t]{3}{*}{ Plantago spp. } & Wägerich, Wägerech & Plantago lanceolata L. & $\begin{array}{l}\text { Spitzwägerich, } \\
\text { Spitzwägerech, } \\
\text { Spitzwegerich, } \\
\text { Wägerich mit } \\
\text { länge Bletter }\end{array}$ & $\begin{array}{l}0809042-2 \\
0908151-1 \\
0908151-2\end{array}$ \\
\hline & & Plantago major L. s.l. & $\begin{array}{l}\text { Breitwägerich, } \\
\text { Breitwägerech, } \\
\text { Wägerich mit } \\
\text { churze Bletter }\end{array}$ & $\begin{array}{l}0809041-3 \\
0908152-3 \\
0908152-4\end{array}$ \\
\hline & & Plantago media L. & Mittlerer Wegerich & \\
\hline \multirow[t]{9}{*}{ Poaceae } & $\begin{array}{l}\text { Gras, Grasarte, } \\
\text { Greser, Schmäle, } \\
\text { Schmale, Schmaale }\end{array}$ & Agrostis stolonifera $\mathrm{L}$. & Struussgras & \\
\hline & & $\begin{array}{l}\text { Alopecurus myosuroides } \\
\text { HUDS. }\end{array}$ & Acherfuchsschwanz & \\
\hline & & Alopecurus pratensis $\mathrm{L}$. & $\begin{array}{l}\text { Fuchsschwanz, } \\
\text { Wisefuchsschwanz }\end{array}$ & \\
\hline & & $\begin{array}{l}\text { Anthoxanthum } \\
\text { odoratum L. }\end{array}$ & $\begin{array}{l}\text { Ruchgras, } \\
\text { Gruchgras, } \\
\text { Geruchgras }\end{array}$ & \\
\hline & & $\begin{array}{l}\text { Arrhenatherum elatius } \\
\text { (L.) J. \& C. PRESL }\end{array}$ & $\begin{array}{l}\text { Fromentau, } \\
\text { Fromental, } \\
\text { französisches } \\
\text { Reigras }\end{array}$ & $\begin{array}{l}0809102-1 \\
0809102-2\end{array}$ \\
\hline & & Briza media L. & Zittergras & $\begin{array}{l}0907084-3 \\
0907084-4\end{array}$ \\
\hline & & $\begin{array}{l}\text { Bromus erectus HUDS. s. } \\
\text { str. }\end{array}$ & Trespe, Mareilihoor & $\begin{array}{l}0810077-1 \\
0810077-2\end{array}$ \\
\hline & & Bromus hordeaceus L. & $\begin{array}{l}\text { weichi Treschpe, } \\
\text { weichi Träschpe }\end{array}$ & \\
\hline & & Cynosurus cristatus L. & Kammgras & \\
\hline
\end{tabular}


Dactylis glomerata $\mathrm{L}$.

Chnougras,

Chnoulgras,

Chnaulgras,

Chnöiugras,

chmale

Elymus repens (L.) Riischgras, Quecke, 081007 6-1

GOULD Spitzgras, 081007 6-2

Schnüergras

Festuca spp.

(Schwingu

Schwingel)

Festuca arundinacea

SCHREB. s.l.

$\begin{array}{ll}\text { Festuca pratensis } & \text { Wiseschwingu, } \\ \text { HUDS. s.l. } & \text { Wiseschwingel, } \\ & \text { Wiesenschwingel, } \\ & \text { Schwingu }\end{array}$

Festuca rubra L. agg. Rotschwingu

Holcus lanatus L.

wolliges Honiggras, $0809104-3$ wolligs Honiggras, $0809104-4$ Wollgras

Lolium perenne L./L

Reigras

multiflorum LAM. var.

westerwoldicum WITTM.

Nardus stricta L.

Burschtgras

Phleum pratense agg.

Poa spp.

Timotee

Rischpegras,

Spitzgras,

Fänschgras

\begin{tabular}{|c|c|c|}
\hline $\begin{array}{l}\text { Lolium multiflorum } \\
\text { LAM. }\end{array}$ & $\begin{array}{l}\text { italiänisches } \\
\text { Reigras, italiänisch } \\
\text { Reigras }\end{array}$ & $\begin{array}{l}0809104-1 \\
0809104-2\end{array}$ \\
\hline $\begin{array}{l}\text { Lolium multiflorum } \\
\text { LAM. var. } \\
\text { westerwoldicumWITTM }\end{array}$ & $\begin{array}{l}\text { Westerwoldisches } \\
\text { Reigras }\end{array}$ & \\
\hline Lolium perenne L. & $\begin{array}{l}\text { Änglisch Reigras, } \\
\text { änglisches Reigras, } \\
\text { änglisches } \\
\text { Reegras, Reigras, } \\
\text { Rischgras, } \\
\text { Weidlgras, } \\
\text { französisches } \\
\text { Reigras }\end{array}$ & $\begin{array}{l}0907308-3 \\
0907308-4\end{array}$ \\
\hline Poa annua $\mathrm{L}$. & $\begin{array}{l}\text { Eijärigs } \\
\text { Rischpegras, } \\
\text { Fänschgras, } \\
\text { Spitzgras }\end{array}$ & $\begin{array}{l}0810071-1 \\
0810071-2\end{array}$ \\
\hline Poa pratensis agg. & $\begin{array}{l}\text { Wiserischpegras, } \\
\text { meerjärigs } \\
\text { Rischpegras, } \\
\text { Spitzgras }\end{array}$ & \\
\hline
\end{tabular}




\begin{tabular}{|c|c|c|c|c|c|}
\hline $\begin{array}{l}\text { Polygonatum multiflorum } \\
\text { (L.) ALL. }\end{array}$ & Salomonssigu & $0907301-130907301-14$ & & & \\
\hline Polygonum bistorta L. & $\begin{array}{l}\text { Zaanbürschtli, } \\
\text { Zangbürschtli, } \\
\text { Wisechnöterich }\end{array}$ & $0907302-30907302-4$ & & & \\
\hline Polygonum persicaria $\mathrm{L}$. & $\begin{array}{l}\text { pfirsichblättriger } \\
\text { Knöterich, } \\
\text { Chnöterich, } \\
\text { Knöterich }\end{array}$ & $0809304-70809304-8$ & & & \\
\hline \multirow[t]{3}{*}{$\begin{array}{l}\text { Polypodiaceae (Dryopteris } \\
\text { spp./ Athyrium/ Oreopteris/ } \\
\text { Pteridium) }\end{array}$} & Farn, Farne & $0809302-1$ & $\begin{array}{l}\text { Dryopteris filix-mas (L.) } \\
\text { SCHOTT/Athyrium filix- } \\
\text { femina (L.) ROTH/ } \\
\text { Oreopteris limbosperma } \\
\text { (ALL.) HOLUB }\end{array}$ & $\begin{array}{l}\text { Wurmfarn, } \\
\text { Stockfarn, } \\
\text { Fäderfarn }\end{array}$ & 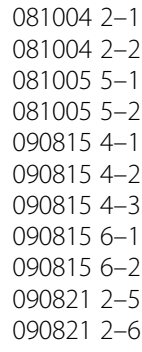 \\
\hline & & & $\begin{array}{l}\text { Pteridium aquilinum (L.) } \\
\text { KUHN }\end{array}$ & $\begin{array}{l}\text { Adlerfarn } \\
\text { Stängufarn angere } \\
\text { Farn }(><\text { Stockfarn) }\end{array}$ & $\begin{array}{l}0810055-7 \\
0810055-8 \\
0908157-1 \\
0908157-2\end{array}$ \\
\hline & & & undef. & $\begin{array}{l}\text { chliises Farn im } \\
\text { Wald }\end{array}$ & \\
\hline Populus tremula $\mathrm{L}$. & $\begin{array}{l}\text { Zitterpapple, Papple, } \\
\text { Eschpe, Espe }\end{array}$ & & & & \\
\hline Potentilla anserina $\mathrm{L}$. & Gänsefingerkraut & & & & \\
\hline $\begin{array}{l}\text { Potentilla erecta (L.) } \\
\text { RAEUSCH. }\end{array}$ & Bluetwurz & & & & \\
\hline $\begin{array}{l}\text { Primula elatior (L.) L./ } \\
\text { Primula veris } \mathrm{L} \text {. S.str. }\end{array}$ & $\begin{array}{l}\text { Schlüssublueme, } \\
\text { Schlüssublüemli, } \\
\text { Schlüsseli }\end{array}$ & & Primula elatior (L.) L. & Schlüssublüemli & $\begin{array}{l}0905102-1 \\
0905102-2 \\
0905102-3\end{array}$ \\
\hline Primula veris L. s.str. & Ehrezäicheli & & & & \\
\hline
\end{tabular}


Table 2 Generic, specific and varietal taxa (Continued)

\begin{tabular}{|c|c|c|c|c|c|c|c|}
\hline$\overline{P r u n u s ~ a r m e n i a c a ~} \mathrm{~L}$. & Aprikose & & & & & & \\
\hline \multirow[t]{4}{*}{ Prunus avium L. } & $\begin{array}{l}\text { Chriesi, Chriesboum, } \\
\text { Chriesiboum, } \\
\text { Chriesibaum, } \\
\text { Chirschi, } \\
\text { Chirschiboum, } \\
\text { Chirschibuum, } \\
\text { Chirschboum, } \\
\text { Kirsche }\end{array}$ & & Prunus avium L. & $\begin{array}{l}\text { wiudi Chirschi, } \\
\text { wildi Chriesi }\end{array}$ & $\begin{array}{l}0907301-7 \\
0907301-8\end{array}$ & & \\
\hline & & & Prunus avium L. (cult.) & $\begin{array}{l}\text { Chriesi, } \\
\text { Chriesboum, } \\
\text { Chriesiboum, } \\
\text { Chriesibaum, } \\
\text { Chirschi, } \\
\text { Chirschiboum, } \\
\text { Chirschibuum, } \\
\text { Chirschboum, } \\
\text { Kirsche }\end{array}$ & & $\begin{array}{l}\text { Prunus avium L. (cult. } \\
\text { black varieties) }\end{array}$ & $\begin{array}{l}\text { Schwarzi } \\
\text { Chirschiböim }\end{array}$ \\
\hline & & & & & & $\begin{array}{l}\text { Prunus avium L. (cult. } \\
\text { black variety) }\end{array}$ & Rigi-Chriesi \\
\hline & & & & & & $\begin{array}{l}\text { Prunus avium L. (cult. } \\
\text { red varieties) }\end{array}$ & Roti Chirschiböim \\
\hline Prunus domestica L. & $\begin{array}{l}\text { Zwätschgeboum, } \\
\text { Zwätschgebuum, } \\
\text { Zwätschgebaum, } \\
\text { Zwätschge }\end{array}$ & & & & & & \\
\hline $\begin{array}{l}\text { Prunus domestica L. ssp. } \\
\text { prisca BERTSCH }\end{array}$ & $\begin{array}{l}\text { Ziberliboum, Ziberli, } \\
\text { Ziiberli }\end{array}$ & $0810078-10810078-2$ & & & & & \\
\hline $\begin{array}{l}\text { Prunus domestica L. ssp. } \\
\text { syriaca (BORKH.) JANCH. }\end{array}$ & Mirabelle & & & & & & \\
\hline Prunus insititia $\mathrm{L}$. & $\begin{array}{l}\text { Pflüümliboum, } \\
\text { Pfluumeboum, } \\
\text { Pflüümli, Pfluume, } \\
\text { Zwätschge }\end{array}$ & & & & & & \\
\hline Prunus persica (L.) BATSCH & Pfirsich & & & & & & \\
\hline Prunus spinosa $\mathrm{L}$. & Schwarzdorn & & & & & & \\
\hline $\begin{array}{l}\text { Pseudotsuga menziesii } \\
\text { (MIRB.) FRANCO }\end{array}$ & $\begin{array}{l}\text { Duglasie, Duglase, } \\
\text { Duglas }\end{array}$ & & & & & & \\
\hline $\begin{array}{l}\text { Psilocybe semilanceata (Fr.) } \\
\text { P. KUMM. }\end{array}$ & $\begin{array}{l}\text { Psilos, magic } \\
\text { mushrooms }\end{array}$ & & & & & & \\
\hline Pyrus communis L. & $\begin{array}{l}\text { Bireboum, Birebuum, } \\
\text { Birebaum, Bire, Birne }\end{array}$ & & & & & & \\
\hline
\end{tabular}

Pflüümliboum

Pfluumeboum,

Pflüümli, Pfluume,

Prunus spinosa L.

(MIRB.) FRANCO

Duglasie, Duglase,

black variety

Prunus avium L. (cult. Roti Chirschiböim

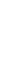


Table 2 Generic, specific and varietal taxa (Continued)

\begin{tabular}{|c|c|c|c|c|c|c|c|c|}
\hline Quercus robur $\mathrm{L}$. & Eiche & $0907301-90907301-10$ & & & & & & \\
\hline \multirow[t]{3}{*}{ Ranunculus spp. } & \multirow[t]{3}{*}{ Hanefuess, Hänifuess } & & $\begin{array}{l}\text { Ranunculus } \\
\text { aconitifolius L. }\end{array}$ & \multicolumn{2}{|l|}{ wiisse Hanefuess } & & & \\
\hline & & & \multirow[t]{2}{*}{$\begin{array}{l}\text { Ranunculus spp. } \\
\text { (flowering yellow) }\end{array}$} & $\begin{array}{l}\text { Hanefuess, gäubi } \\
\text { Ankeblüemli, } \\
\text { Ankeblueme, chliini } \\
\text { gäubi Blüemli }\end{array}$ & $\begin{array}{l}0907306-5 \\
0907306-6 \\
1008011-5\end{array}$ & Ranunculus acris L. s.l. & $\begin{array}{l}\text { angere Hanefuess } \\
(><\text { kriechend), } \\
\text { scharfe Hanefuess }\end{array}$ & $0809042-1$ \\
\hline & & & & & & Ranunculus repens L. & $\begin{array}{l}\text { kriechender } \\
\text { Hanefuess, breite } \\
\text { Hanefuess }\end{array}$ & \\
\hline \multirow[t]{2}{*}{ Raphanus/Sinapis } & \multirow[t]{2}{*}{ (Sänf) } & & $\begin{array}{l}\text { Raphanus } \\
\text { raphanistrum L. }\end{array}$ & Wiisse Sänf & $\begin{array}{l}1008014-5 \\
1008014-6 \\
1008014-7 \\
1008014-8\end{array}$ & & & \\
\hline & & & Sinapis arvensis $\mathrm{L}$. & Gäube Sänf & $\begin{array}{l}1008017-3 \\
1008017-4\end{array}$ & & & \\
\hline $\begin{array}{l}\text { Raphanus sativus L. var. } \\
\text { niger (MILL.) J. KERN }\end{array}$ & \multicolumn{2}{|l|}{ Rättich, Rettech } & & & & & & \\
\hline $\begin{array}{l}\text { Raphanus sativus L. var. } \\
\text { sativus (red varieties) }\end{array}$ & \multicolumn{2}{|l|}{ Radiisli } & & & & & & \\
\hline Rheum rhabarbarum L. & \multicolumn{2}{|l|}{ Rebarbere } & & & & & & \\
\hline $\begin{array}{l}\text { Rhinanthus alectorolophus } \\
\text { (SCOP.) POLLICH }\end{array}$ & $\begin{array}{l}\text { Klappertopf, } \\
\text { Chlappertopf, } \\
\text { Klappergras }\end{array}$ & 090708 3-1 090708 3-2 & & & & & & \\
\hline $\begin{array}{l}\text { Rhododendron ferrugineum } \\
L . / R \text {. hirsutum } L \text {. }\end{array}$ & \multicolumn{3}{|l|}{ Auperose } & & & & & \\
\hline Rhododendron sp. (cult.) & \multicolumn{3}{|l|}{ Rhododendron } & & & & & \\
\hline Ribes rubrum L./R. nigrum L. & Trübeli, Meertrübeli & & Ribes nigrum L. & $\begin{array}{l}\text { Schwarzi Trübeli, } \\
\text { Cassi, Cassis }\end{array}$ & & & & \\
\hline Ribes rubrum L. & $\begin{array}{l}\text { Roti Trübeli, Trübeli, } \\
\text { Meertrübeli, } \\
\text { Johannisbeeri }\end{array}$ & & & & & & & \\
\hline Ribes uva-crispa L. & $\begin{array}{l}\text { Chrusle, Stachubeeri, } \\
\text { Stachelbeere }\end{array}$ & & & & & & & \\
\hline \multirow[t]{2}{*}{ Ribes $x$ nidigrolaria } & \multirow{2}{*}{\multicolumn{2}{|c|}{ Joschta }} & & & & & & \\
\hline & & & Robinia pseudoacacia L. & $\begin{array}{l}\text { Robinie, fautschi } \\
\text { Akazie }\end{array}$ & & & & \\
\hline \multirow[t]{2}{*}{ Rosa spp. } & \multirow[t]{2}{*}{ Rose } & & Rosa spp. (cult.) & Rose, Roseböimli & & & & \\
\hline & & & $\begin{array}{l}\text { Rosa spp. (wild, e. g. R. } \\
\text { canina L., R. pendulina L.) }\end{array}$ & $\begin{array}{l}\text { wiudi Rose, } \\
\text { Hagrose, }\end{array}$ & & & & \\
\hline
\end{tabular}


Table 2 Generic, specific and varietal taxa (Continued)

Hagrööseli,

Hagbuttestruuch,

Hagebutte (fruits)

$\begin{array}{ll}\text { Rosmarinus officinalis L. } & \text { Rosmarin, Rosmarii } \\ \text { Rubus fruticosus agg. } & \text { Brombeeri, } \\ & \text { Brommerli, Brumeli, } \\ & \text { Brummbeeri }\end{array}$

Rubus fruticosus agg. (cult.) Brombeeri,

Brommerli,

Brummbee

Himbeeri, Himbeere, 090730 1-5090730 1-6

$1008012-31008012-4$

Himpeli, Himpi, Hinti

Rudbeckia hirta L. Rudbeckia

Rumex acetosa $\mathrm{L}$

Surampfer

Surampfere,

Sauerampfer

Suurchrut,

Guggersuur

Rumex obtusifolius L.

Blacke, Blacki,

Wiseblacke,

Dittiblacke,

Salix spp.

Salvia spp.

Wide, Weide

Saubei, Salbei,

Saubine, Salbine

090730 4-11 090730 4-12
Rubus fruticosus agg.

fruits: wüudi

plant: Dörn

Brammertörn,

Brambeeritörn,

Brombeeritörn

Brumelidörn,

Dornbüsch
100801 3-1 100801 5-7

Salvia nemorosa $\mathrm{L}$.

Salvia officinalis $\mathrm{L}$.

Salvia officinalis L. "Tricolor"

Salvia pratensis $\mathrm{L}$.

Salvia splendens

SELLOW ex ROEM. \&

SCHULT.

Sambucus nigra L. 
Table 2 Generic, specific and varietal taxa (Continued)

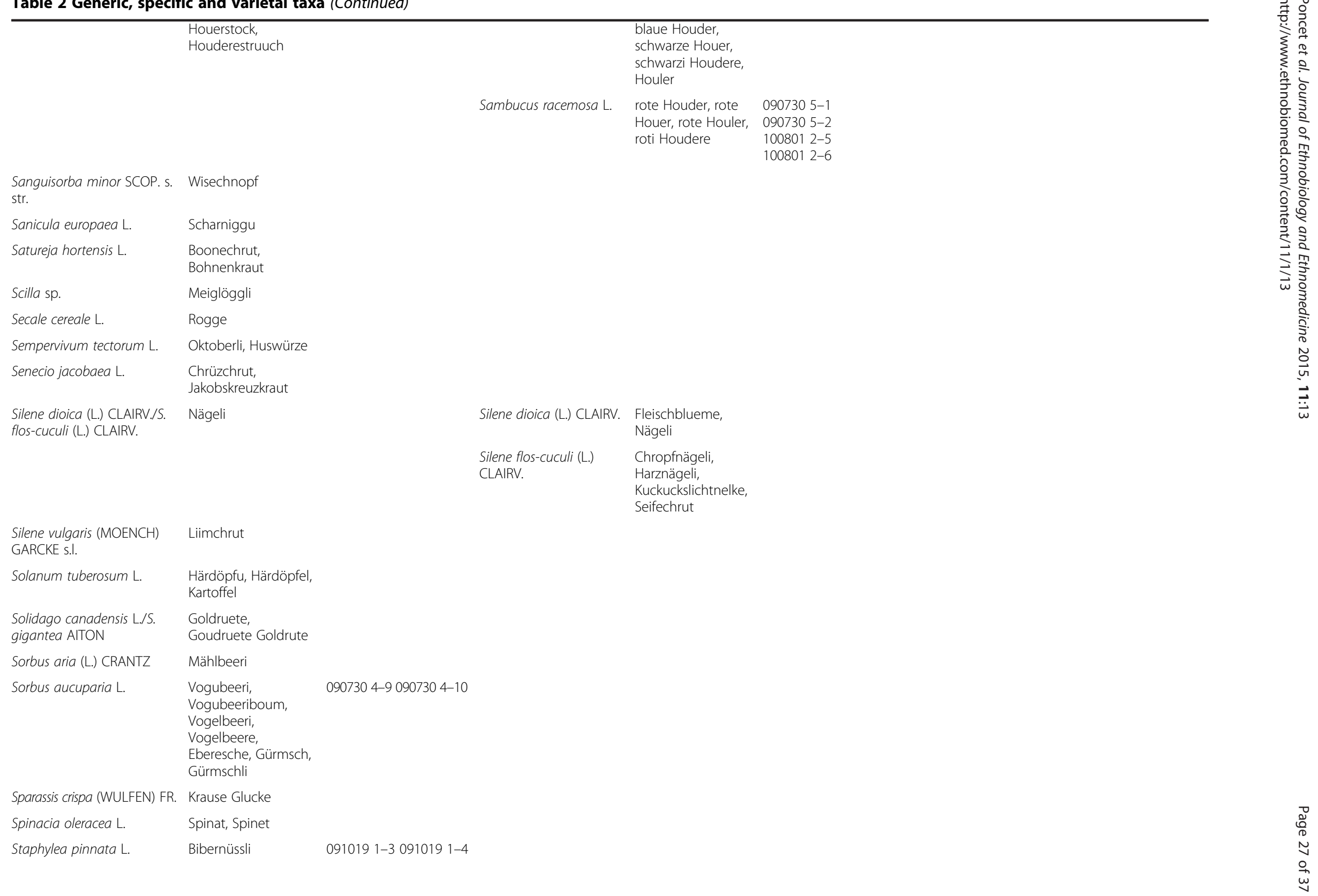


Table 2 Generic, specific and varietal taxa (Continued)

\begin{tabular}{|c|c|c|c|c|c|}
\hline$\overline{\text { Stellaria media (L.) VILL. }}$ & $\begin{array}{l}\text { Hüenerdarm, } \\
\text { Hüenderdarm, } \\
\text { Vogumiere, } \\
\text { Vögelichrut }\end{array}$ & $0809305-30809305-4$ & & & \\
\hline $\begin{array}{l}\text { Symphoricarpos albus (L.) S. } \\
\text { F. BLAKE }\end{array}$ & $\begin{array}{l}\text { Struuch mit wiisse } \\
\text { Bböueli }\end{array}$ & & & & \\
\hline Symphytum officinale L. & $\begin{array}{l}\text { Wallwurz, Wauwürze, } \\
\text { Beinwell }\end{array}$ & & & & \\
\hline Syringa vulgaris $\mathrm{L}$. & Flider & & & & \\
\hline Tagetes sp. & Tagetes & & & & \\
\hline Tanacetum vulgare $\mathrm{L}$. & Reinfarn & $\begin{array}{l}0907309-50907309-6 \\
1008016-11008016-1\end{array}$ & & & \\
\hline Taraxacum officinale s.l. agg. & $\begin{array}{l}\text { Söiblueme, } \\
\text { Süiblueme, } \\
\text { Löwezaan, } \\
\text { Chrottepösche }\end{array}$ & & & & \\
\hline Taxus baccata $\mathrm{L}$. & Eibe & $1003281-8$ & & & \\
\hline Thuja occidentalis L. & Thujahaag & & & & \\
\hline Thymus spp. & Timian, Thümian & $\begin{array}{l}081005 \text { 6-1 } 081005 \text { 6-2 } \\
0908153-60908153-7\end{array}$ & $\begin{array}{l}\text { Thymus serpyllum } \mathrm{L} \text {. } \\
\text { agg. }\end{array}$ & $\begin{array}{l}\text { wiude Tümian, } \\
\text { wüude Timian }\end{array}$ & \\
\hline Thymus vulgaris $\mathrm{L}$. & $\begin{array}{l}\text { Tümian, } \\
\text { Gartentümian }\end{array}$ & & & & \\
\hline $\begin{array}{l}\text { Thymus vulgaris L. } \\
\text { "fragrantissimus" }\end{array}$ & Orangschetimian & & & & \\
\hline \multirow[t]{2}{*}{$\begin{array}{l}\text { Tilia platyphyllos SCOP./ T. } \\
\text { cordata MILL. }\end{array}$} & $\begin{array}{l}\text { Linde, Lindeboum, } \\
\text { Lindebaum, Linge; } \\
\text { flowers: Lindeblüete, } \\
\text { Lindebluescht }\end{array}$ & 090702 3-1 090702 3-2 & Tilia cordata MILL. & Winterlinde & \\
\hline & & & Tilia platyphyllos SCOP. & Summerlinde & \\
\hline Tradescantia sp. & Gottesauge & & & & \\
\hline Tragopogon pratensis L. s.l. & Bockbart & & & & \\
\hline \multirow[t]{2}{*}{$\begin{array}{l}\text { Trifolium/Lotus/Medicago/ } \\
\text { Oxalis }\end{array}$} & Chlee & & Lotus corniculatus agg. & $\begin{array}{l}\text { Gäubchlee, } \\
\text { Gälchlee, gäube } \\
\text { Chlee, Schotechlee, } \\
\text { Steichlee, } \\
\text { Steechlee, } \\
\text { Steinklee, } \\
\text { Hornchlee }\end{array}$ & $\begin{array}{ll}090702 & 2-1 \\
090702 & 2-2 \\
090708 & 2-3 \\
090708 & 2-4\end{array}$ \\
\hline & & & $\begin{array}{l}\text { Medicago lupulina L./ } \\
\text { Trifolium campestre } \\
\text { SCHREB. }\end{array}$ & $\begin{array}{l}\text { Hopfechlee, } \\
\text { Gäubchlee }\end{array}$ & $\begin{array}{l}0907071-7 \\
0907071-8\end{array}$ \\
\hline
\end{tabular}


Table 2 Generic, specific and varietal taxa (Continued)

\begin{tabular}{|c|c|c|c|c|c|}
\hline & & & Oxalis acetosella $\mathrm{L}$. & $\begin{array}{l}\text { Suurchlee, } \\
\text { Waudchlee, } \\
\text { Hasechlee }\end{array}$ & $\begin{array}{l}0905103-1 \\
0905103-2 \\
0905103-3 \\
0907084-5 \\
0907084-6\end{array}$ \\
\hline & & & Trifolium alexandrinum L. & Alexandrinerchlee & \\
\hline & & & Trifolium hybridum L. s.str. & Baschtardchlee & \\
\hline & & & Trifolium incarnatum L. & Inkarnatchlee & \\
\hline & & & $\begin{array}{l}\text { Trifolium pratense L. s.l./ } \\
\text { T. medium L./ T. repens } \mathrm{L} \text {. }\end{array}$ & $\begin{array}{l}\text { Chleeblettli, } \\
\text { Chleeblüemli, } \\
\text { vierbletterigs } \\
\text { Chleeblatt }\end{array}$ & \\
\hline & & & $\begin{array}{l}\text { Trifolium pratense L. S. } \\
\text { str. / T. medium L. }\end{array}$ & $\begin{array}{l}\text { Rotchlee, } \\
\text { Chleeblüemli, } \\
\text { Mattechlee }\end{array}$ & $\begin{array}{l}0907025-5 \\
0907025-6\end{array}$ \\
\hline & & & Trifolium repens $\mathrm{L}$. & $\begin{array}{l}\text { Wiss-Chlee, } \\
\text { Weissklee, } \\
\text { Chleeblüemli }\end{array}$ & $\begin{array}{l}0809041-4 \\
0907025-3 \\
0907025-4\end{array}$ \\
\hline Triticum aestivum L. & $\begin{array}{l}\text { Weize, Winterweize, } \\
\text { Wäize }\end{array}$ & & & & \\
\hline Triticum spelta $\mathrm{L}$. & Dinku, Dinkel, Chorn & & & & \\
\hline Trollius europaeus L. & $\begin{array}{l}\text { Ankebäueli, } \\
\text { Ankebäui, Töni, } \\
\text { Moosbouele }\end{array}$ & & & & \\
\hline Tropaeolum majus $\mathrm{L}$. & Stigüferli, Kapuzinerli & & & & \\
\hline Tulipa sp. (cult.) & Tuupe, Tulpe & & & & \\
\hline Tussilago farfara $\mathrm{L}$. & $\begin{array}{l}\text { Zitröseli, Zitteröseli, } \\
\text { Windröseli, Huflattich }\end{array}$ & $0905101-10905101-2$ & & & \\
\hline Ulmus glabra HUDS. & Uume, Ulme & & & & \\
\hline Urtica dioica $\mathrm{L}$. & $\begin{array}{l}\text { Brönessle, Bränessle, } \\
\text { Nessle }\end{array}$ & & & & \\
\hline \multirow[t]{2}{*}{$\begin{array}{l}\text { Vaccinium myrtillus L./ V. } \\
\text { corymbosum L. }\end{array}$} & $\begin{array}{l}\text { Höibeeri, Heidubeeri, } \\
\text { Heidelbeeri, } \\
\text { Heidelbeere }\end{array}$ & & Vaccinium corymbosum L. & Heidubeeri & \\
\hline & & & Vaccinium myrtillus L. & $\begin{array}{l}\text { Höibeeri, } \\
\text { Höibeeristude, } \\
\text { Höibeeri im Waud, } \\
\text { wiudi Höibeeri }\end{array}$ & $\begin{array}{l}0908182-1 \\
0908182-2 \\
0908182-3 \\
1008016-5 \\
1008016-6\end{array}$ \\
\hline Vaccinium vitis-idae $\mathrm{L}$. & Fuchsbeeri & $\begin{array}{l}090815 \text { 9-1 } 0908159-2 \\
090815 \text { 9-3 }\end{array}$ & & & \\
\hline
\end{tabular}


Table 2 Generic, specific and varietal taxa (Continued)

\begin{tabular}{|c|c|c|c|c|c|c|}
\hline Valeriana officinalis agg. & Baudrian & & & & & \\
\hline Verbascum spp. & $\begin{array}{l}\text { Chünigs-Cherze, } \\
\text { Chönigs-Cherze, } \\
\text { Wuublüemli, } \\
\text { Wuublueme, } \\
\text { Wolleblüemli }\end{array}$ & & & & & \\
\hline $\begin{array}{l}\text { Veronica chamaedrys L./V. } \\
\text { filiformis SM./ V. persica } \\
\text { POIR }\end{array}$ & $\begin{array}{l}\text { Chatzenöögli, } \\
\text { Chatzenöigli, } \\
\text { Chatzeöögli, } \\
\text { Chatzeöigli, } \\
\text { Chatzenouge, } \\
\text { Ehrenpreis }\end{array}$ & & & & & $\begin{array}{l}0907024-3090702 \\
4-4090702 \text { 4-7 } \\
0907024-8100801 \\
11-1 \quad 100801 \quad 11-2 \\
100801 \quad 11-3\end{array}$ \\
\hline Veronica officinalis L. & Eerepriis & & & & & \\
\hline Viburnum spp. & Schneebau & & Viburnum lantana L. & $\begin{array}{l}\text { Wüude Schnebau, } \\
\text { Vogubeeri, wolliger } \\
\text { Schnebau }\end{array}$ & & \\
\hline & & & Viburnum opulus L. & $\begin{array}{l}\text { angere Schnebau } \\
(><\text { wolliger), } \\
\text { Schneebau }\end{array}$ & $\begin{array}{ll}090730 & 1-3 \\
090730 & 1-4\end{array}$ & \\
\hline Vicia cracca L. s.I.JV. sepium L. & $\begin{array}{l}\text { en Art Ärbsgwächs, } \\
\text { Wicke }\end{array}$ & $0907071-10907071-2$ & & & & \\
\hline Vinca major L. & Stritte & & & & & \\
\hline Viola spp. & Veieli & $0905104-10905104-2$ & Viola odorata L. & Veieli & & \\
\hline & & & $\begin{array}{l}\text { Viola reichenbachiana } \\
\text { BOREAU/V. riviniana } \\
\text { RCHB. }\end{array}$ & $\begin{array}{l}\text { Tubechropf, } \\
\text { Hundsveieli }\end{array}$ & & \\
\hline Viola tricolor agg. & $\begin{array}{l}\text { Stifmüeterli, } \\
\text { Stöifmüeterli }\end{array}$ & & & & & \\
\hline Viscum album L. s.l. & $\begin{array}{l}\text { Mischtle, Mischteler, } \\
\text { Mistel }\end{array}$ & & & & & \\
\hline Vitis vinifera $\mathrm{L}$. & Trube, Räbe & & & & & \\
\hline$x$ Triticosecale & Tritical, Triticau & & & & & \\
\hline Zea mays L. & Meis, Mäis & & & & & \\
\hline
\end{tabular}


Table 3 General purpose higher order taxa

\begin{tabular}{|c|c|c|c|c|c|}
\hline \multicolumn{2}{|l|}{ Life-form taxa } & \multicolumn{2}{|l|}{ Intermediate taxa (1st order) } & \multicolumn{2}{|c|}{ Intermediate taxa (2nd order) } \\
\hline Local name & English term & Local name & English term & Local name & English term \\
\hline \multirow[t]{6}{*}{ Boum } & tree & & & & \\
\hline & & Naduboum, Nadelboum, Tanne & Conifer & & \\
\hline & & Loubboum & Deciduous tree & & \\
\hline & & Obschtboum & Fruit-tree & & \\
\hline & & & & Steiobscht & Stone fruits \\
\hline & & & & Chärnobscht & Pomaceous fruits \\
\hline \multirow[t]{2}{*}{ Stude, Struuch } & Bush, shrub & & & & \\
\hline & & Beeristude & Berry bush & & \\
\hline Gras, Greser & Grass & & & & \\
\hline Chrüttli, Chrütter & Herb & & & & \\
\hline Blüemli, Blueme & Flower & & & & \\
\hline Piuz, Pilz & Mushroom & & & & \\
\hline
\end{tabular}

often mentioned trait was the life-form, followed by fruit, leave and flower (Figure 2).

The most important use relevant for the sorting of plants was edibility (Figure 3). Berries were put together because they are used for desserts, jam and syrup, fruit trees because of their similar use for eating, juice and schnapps, wild or garden herbs because of their use in salads, as spices or for herbal tea. Other groups contained fodder plants for the cattle, medicinal or ornamental plants. Tree groups were sometimes explained with the argument that their wood can be used for construction or as firewood. In 37 cases the formed group was declared as "plants of no use" or "useless weeds". They were also noted as use-groups in the sense that they are defined by non-use.

During the pile sorting task 11 habitats were used to explain the groups (Figure 4). The most often mentioned habitats "meadow/pasture", "forest" and "garden" were further divided into sub-habitats. As sub-habitats for meadows and pastures appeared: extensive (nutrient poor) meadow, intensive (nutrient rich) meadow, artificial meadow, forest meadow, meadow with sour soil, wet pasture. Sub-habitats of the forest were: Lothar-forest (patches of forest where most of the trees were knocked over in 1999 by the storm "Lothar") and the differentiation into trees and brushwood. Sub-habitats of the garden were: "Pflanzblätz" (a vegetable or fibre crop plot in some distance to the farm) and the differentiation into plants at the fence, in the path and as boundary.

Habitats additionally mentioned during the semistructured interviews but not used for the sorting task were: field, alpine pasture, brookside, pond and "places where many people walk".

\section{Cultural domain analysis of the pile sorting}

Cultural Consensus Analysis did not detect significant variation among the informants (pseudo-reliability 0.990, first eigenvalue ratio 36.256) and the cluster diagram reveals six major groups (Figure 5). The groups are indicated with the

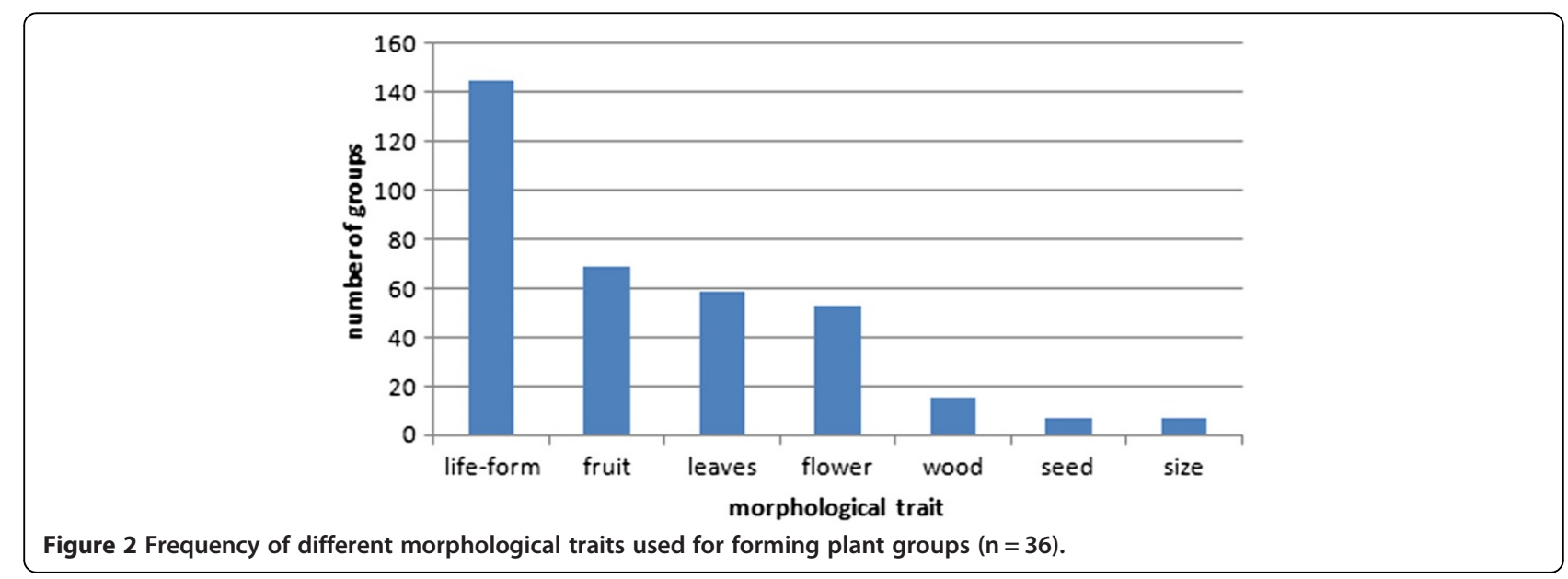




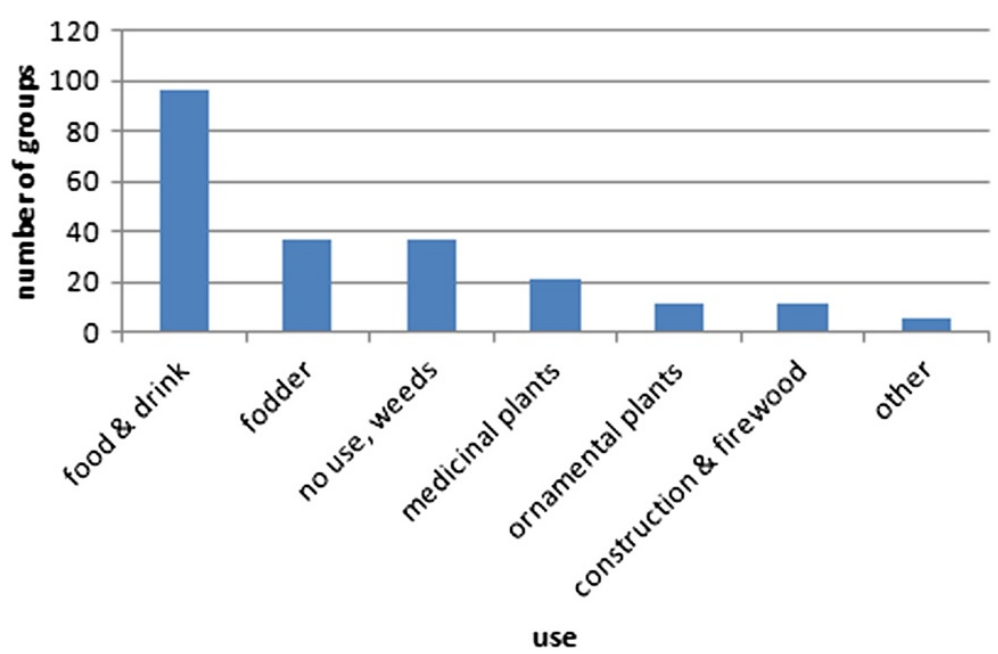

Figure 3 Frequency of different types of uses relevant for forming plant groups $(n=36)$.

name generally given by the informants: grasses, meadow plants/herbs/flowers, trees, weeds, shrubs/hedges, and berries.

Grasses are a well-defined, clearly separated group including the four most valuable forage grasses. It was sometimes split into clover (Trifolium repens, $T$. pratense) and grasses (Dactylis glomerata, Lolium perenne).

The meadow-group can be divided into three main subgroups. The first subgroup contains plants described as "not that good grasses" or "fill-ins": Ranunculus spp., Rumex acetosa, Plantago major, P. lanceolata. They were by most informants perceived as neither good nor bad. The second subgroup contains the tea herbs Alchemilla spp., Mentha piperita and Salvia officinalis, the third subgroup the flowers Bellis perennis, Leucanthemum vulgare, Primula elatior/veris, Taraxacum officinale and Helianthus annuus. The three subgroups overlap one another and some plants were also put in other groups as the "weeds" or the "garden plants" (which do not appear as group in the cluster analysis). This explains the low coherence within the group.

Trees are a very well defined group, which is split into three subgroups. The first contains the fruit-trees pear, apple, prune and cherry (Pyrus communis, Malus domestica, Prunus domestica, P. avium). The second contains the two conifers Abies alba and Picea abies, which supply also the best timber of the region. The third subgroups contains the deciduous trees of the forest (Betula pendula, Acer pseudoplatanus, Fagus sylvatica, Quercus robur, Fraxinus excelsior, Tilia spp.), where Tilia is weakly associated. The lime does not grow wild in the region, but is often planted next to the house or on top of hills. It was often put alone or together with the fruit trees.

Weeds are a small group consisting of Urtica dioica, Rumex obtusifolius, Polypodiaceae, and Cirsium spp. They are closely observed and eventually weeded. The stinging nettle Urtica dioica is rather weakly associated, because it was often put together with tea herbs.

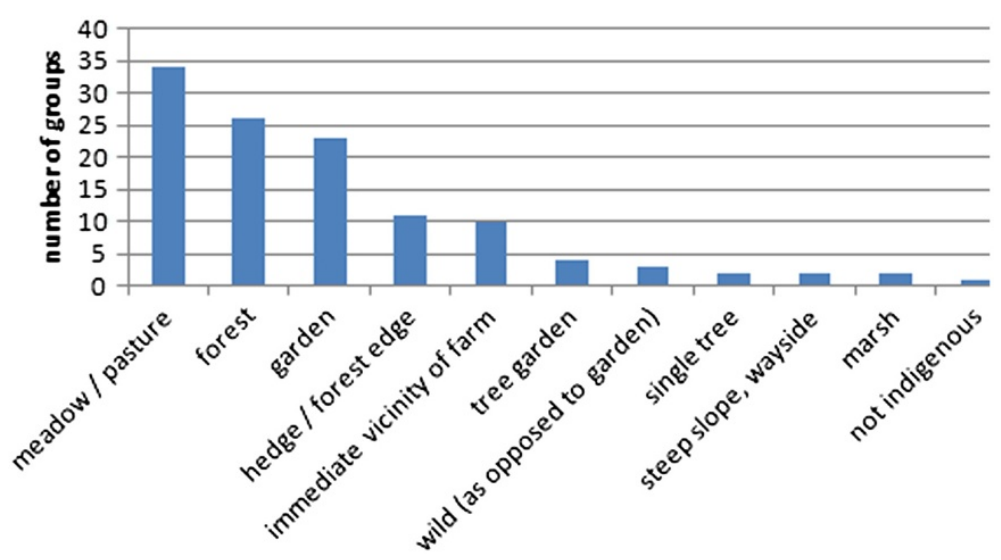

habitat

Figure 4 Frequency of habitats relevant for forming plant groups $(n=36)$. 


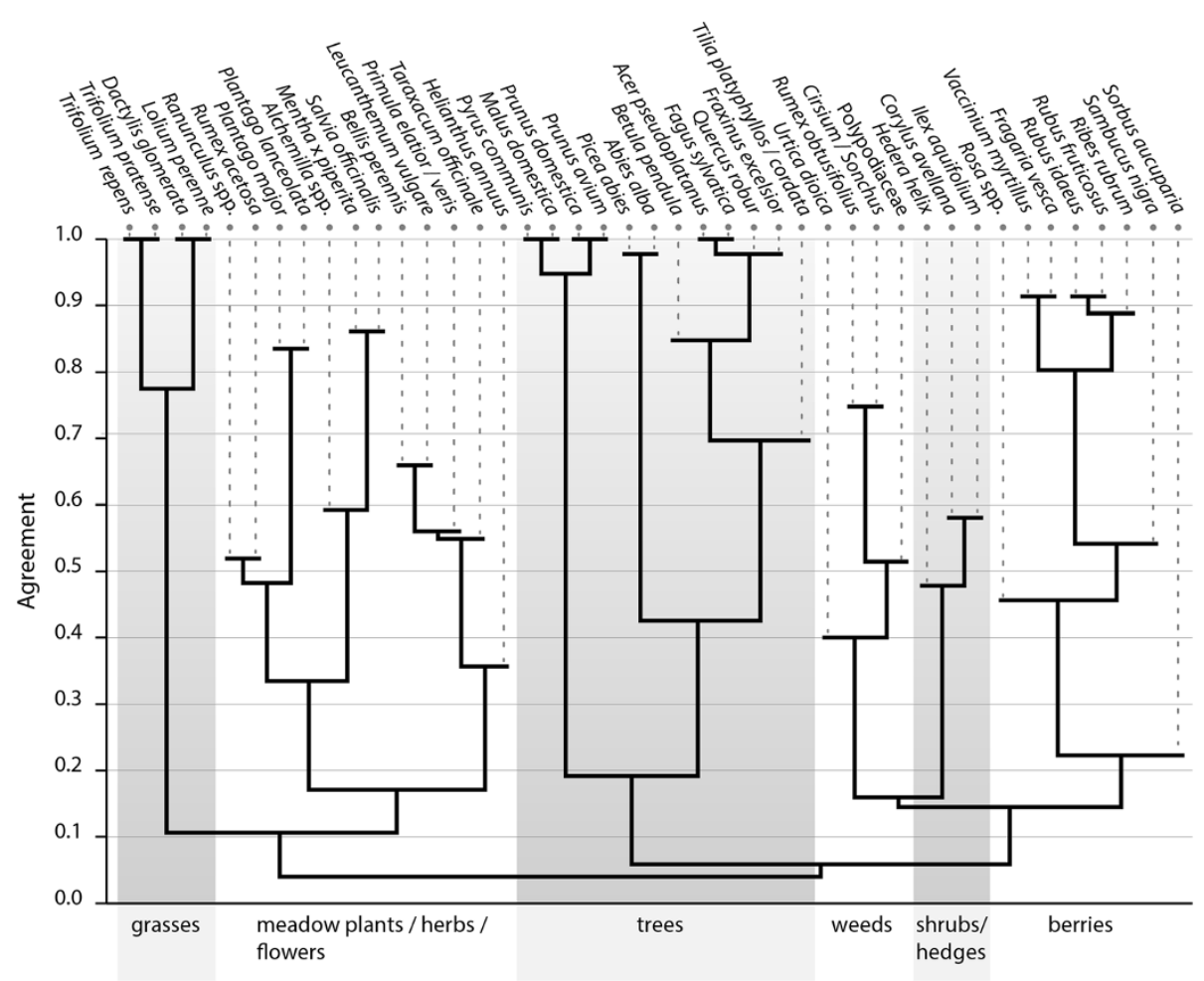

Figure 5 Anthropac cluster analysis of the 36 pile sortings, groups indicated with generally given names $(n=36)$.

The shrubs and hedges group contains Hedera helix, Corylus avellana and Ilex aquifolium. The ivy with its special growth form was difficult to group for the informants. It was often added only at the end of the task to the group which seemed most appropriate e.g. ornamental plants, hedges or the undergrowth of the forest. This group contained often also Sambucus, Rosa and Sorbus. But these three species were apparently more often defined by their fruits and appear therefore in the last group of the berries.

The berries group is defined by species with small, edible fruits, which are used to make jam and syrups: Rosa spp., Vaccinium myrtillus, Fragaria vesca, Rubus idaeus, Rubus fruticosus, Ribes rubrum, and Sambucus nigra. The rowan (Sorbus aucuparia) is weakly associated because although its fruits look similar to other berries, they are often supposed to be poisonous.

\section{Discussion}

\section{Folkbotanical classification}

The folkbotanical classification system of farmers' families in the Napf region shows similar numbers of known plant taxa as those of indigenous societies all over the world [1]. The observed shallow taxonomic hierarchy with few higher order and many lower order taxa (generic, specific) is also typical for folkbotanical classification systems [1]. For the most important rank, the generic species, Berlin [1] extracted numbers from 17 ethnobotanical studies, ranging from 137 to 956 (80\% monotypical), with traditional noncultivators at the lower, and traditional cultivators at the upper end. With 316 generic taxa (85.4\% monotypical), the present study lies in the lower range of the "traditional cultivators". Typical for "traditional cultivators" is also the high amount of sub-generic taxa (e.g., different salads or forage grasses in our case), which is suspected to be driven by cultivation and related close observation of the plants.

The assignment of a taxon to a rank is generally difficult and prone to subjectivity. One of the encountered problems were primary names of specific or subspecific taxa. Primary, often simple names as "thistle" or "hazel" are usually a characteristic of generic taxa. There are, however, a few exceptions. A specific taxon may bear a primary name, if it is the prototype of the respective generic taxon [1], p.29. In our study, the generic taxon "Hasle" (Corylus avellana) is split into the two specific taxa "Hasle" and "Zierhasle", whereby the former is the prototype and the latter a garden form of Corylus avellana. Abbreviation of complex specific or subspecific names may also occur [1], p.29. Our generic "Gras" (Poaceae) contains among many specifics of the type "x-gras" also the specific "Timotee" (Phleum pratense), which is an abbreviation of "Timoteegras". We assigned also "Fuchsschwanz" (Alopecurus spp.), 
"Trespe" (Bromus erectus) and "Goudhaber" (Trisetum flavescens) to the generic "Gras", although they lack the label "-gras" completely. But they were always listed or mentioned together with other grasses, never alone and in the case of "Goudhaber" - never together with "Haber" (Avena sativa). It is also important to keep in mind, that plant names may have very different origins. Most of the grasses were listed and known only by men, especially by farmers, who had completed an agricultural school. From their training they knew the formal names of the grasses and pronounced them often in High German. Recently imported plant names like these cannot be expected to reflect a local classification system. The same is true for many garden plants, which often bear fanciful names.

The above mentioned "Salat" is another example of a folk generic containing several specifics which lack the epithet "-salat" and bear primary names. It could be argued that "Gras" and "Salat" are intermediate and, especially in the case of the salads, use-based taxa. We decided nevertheless to rank them as generics, because it needs expert knowledge to differentiate between the respective specifics. Less knowledgeable persons described the different specific taxa all as "Gras" or "Salat". Furthermore, both names appeared frequently in freelists, where intermediate taxa are uncommon. Nevertheless, many of the generic, specific and subspecific taxa need further investigation to confirm the assigned rank.

The taxa of the intermediate level, which are after Berlin often covert, not named taxa [2], p. 26, were the most difficult to define and are certainly not complete. This is partly also due to the study design. While the open setting of the sorting task allowed identifying different important aspects of plant classification such as morphology, use and ecology (see below), a successive pile sorting [29], for example, would probably have forced the interviewees to classify in a more consequent and hierarchical manner.

\section{Plant knowledge}

Several environmental education studies from industrialized countries show difficulties of young people to list plants. In Switzerland, over 6000 children and adolescents between the age of 8 and 16 listed on average five plants out of their immediate environment [11]. In Germany, only $14 \%$ of 3000 school leavers were able to list eight native wildflowers [36]. In South Carolina, USA, thirty-one 18-22-year-old college students freelisted in different local plant domains on average 9.0 crops, 8.4 trees, 5.4 garden flowers, 1.9 vines, 1.7 wildflowers/weeds and 1.4 grasses, so in total on average 27.8 plants [13]. Still in South Carolina, eleven 9-12-year-old children freelisted on average 30.9 plants in 10 different plant categories like trees, flowers, garden plants, shrubs etc. [37]. In Ajo, a rural town in Arizona, USA, 110 students between the ages of 12 and 20 freelisted on average five and in total 85 plants when asked to "name all of the plants that you know" [12].

In the Napf-region we found higher numbers of freelisted plants. Adults above 20 years listed on average 54 plants and even children and adolescents up to 20 years old listed on average 25 plants and in total 179 different plants. The average number given by children and adolescents is lower than in the two studies of South Carolina, but it was the outcome of only one freelist question. As freelists tend to be more complete the more focused the domain is [38], our number would probably be higher if we would have included more than one freelist question. The very unspecific life-form term "tree", which was frequently mentioned in the above cited studies, did never appear in our freelists. "Grass" on the other hand was often mentioned, mostly by women and children, but meaning then only species of the families Poaceae, Cyperaceae and Juncaceae. It seems that even in industrialized countries, a rural population of small-scale farmers has enough direct contact with the surrounding environment to keep a respectable knowledge about plants.

While the "devolution" of plant knowledge seems less pronounced among rural people, it is still difficult to estimate how much formerly held knowledge has been eroded or changed. For example, the number of plants listed by the Napf children is modest compared to the 43 plant species known by 9 year old Tzeltal Maya children [39], although the difference may also be influenced by the plant diversity of the environment. In our data, we could not detect significant differences in the number of listed plants among older and younger adults. There was a significant increase of listed plants around the age of 20, which is probably due to professional specialization of the adults: Most men are educated farmers and most women manage a large homegarden.

A list of vernacular plant names gathered over the last 50 years in the north-eastern part of the Napf-region and the adjacent rural area of the canton of Lucerne (Amt Willisau) contains names of 653 species, split into 301 wild species, 81 crops and 301 fruit trees and garden plants [40]. Compared to our data (298 wild species, 213 cultivated plants), Brun-Hool reports much more names of cultivated plants, which might indicate a loss of knowledge in our area. However, while Brun-Hool is a specialist for homegardens, our freelist question was rather directed towards wild growing species, which may explain the above differences [41].

\section{Sorting criteria}

Pile sorting is influenced by the (expertise) knowledge of the interviewee in different plant-related areas. Often different criteria are simultaneously used to explain plant (or fish) sorting $[9,14,16,42]$. The more an individual knows 
about a plant, the less it uses only the most obvious morphological features for its assessment, or as Nolan puts it:

“... ethnobotanical classification is based fundamentally on the recognition of ostensible perceptual features of plants, but progressively guided by the recognition of culturally learned functional attributes." [16] p. 69.

In our case this means that a basic morphological system is superposed by culturally influenced knowledge especially about use and habitat of the plants. Only our youngest informant, an eleven-years-old girl, sorted exclusively by morphology. Possibly she did not master additional knowledge to the same degree as others did. Interestingly, informants tended to switch criteria during one and the same pile sorting task. Apart from the unconstrained question the reason may lie in the fuzziness inherent to any given system. People used a new criterion as soon as a plant was difficult to classify.

The salience of morphology, use and habitat is underlined by other studies: all the three appear in an ethnomycological study from Indonesia and a folkbiological study of fish [43,42], morphology and use in classification studies of trees and medicinal plants [14,16], and morphology and habitat in an environmental education study [44]. A study from the French alps identified 23 "folk biotopes" and states that "folk botanical knowledge (...) is perceptually and practically linked to folk ecological knowledge, represented by the set of locally perceived higher order units analogous to the scientific ecologists' habitat and biotop community" [17] p. 55. Additional criteria may emerge in other contexts. Seasonal aspects for example were important for sorting plants in a study in the Grosses Walsertal, Austria [45], personal communication as well as in the mentioned fish studies [9,42].

Despite the open sorting task question, Cultural Consensus Analysis showed a high consensus among the informants and revealed no obvious subgroups or outliers. Even the four persons sorting consequently by a single criterion produced quite similar groups. Thus, the salient groups visible in the cluster analysis were the result of varying considerations. The tree group, for example, was explained mainly by morphology ("they are trees/they look alike"), but also by habitat ("they grow in the forest/ in the tree garden") or use ("we use the wood/the fruits"). The group of the grasses was explained mainly by use ("they are good fodder grasses"), but also by habitat ("they grow in meadows") or morphology ("they are grasses/clovers). The two grasses as well as the two clover species were additionally strongly linked by their names. They were both called "-grass" (-gras: Reigras, Chnoulgras) and "-clover" (-chlee: Wiiss-Chlee, Rotchlee) respectively, which contributed probably to the fact, that they were never separated at all. The berries group was also quite stable, with slight variations: the description "berry" stressed the morphological aspect and included all plants with berry-like fruits, "berries in the garden" or "wild berries/berries in the forest" stressed the habitat and excluded Sambucus nigra, Sorbus aucuparia, Vaccinium myrtillus, Fragaria vesca and Rosa spp. in the first and Ribes rubrum in the second case, while "edible berries / you can make jam of them" stressed the use and excluded Sorbus and also Rosa for most of the people.

Obviously, in our study, "all roads lead to Rome" [14]. With another selection of pile sorting plants, this effect may have been weaker. But the plants forming the stable groups are good examples to demonstrate, that not only form and function $[9,14,16]$, but also habitat and function [17] and, as known from plant ecology, habitat and form may be inherently linked. For example, trees are functional forms and the forest a functional habitat for people cutting and using wood - and forests are characterized by tree species. Grasses and clover are functional forms and meadows functional habitats for people breeding cattle - and meadows are characterized basically by grass species. For the berries, there was a particularly strong link between morphology and use. The term "berry" itself, which in botany is purely morphological, implies in the Napf region almost the edibility of the fruit. A young man explained his berry-group very typically like "Those are berries. Small, round and edible things". He included Sorbus aucuparia, although he was in doubt about its edibility. But the local name "Vogubeeri", literally "bird berry", evocate that it is eaten at least by birds (and links it linguistically to the other berries). Ilex aquifolium, in contrast, appeared never in the berry group. Its local name "Stächpaume" contains no link to the berries and to edibility. The undeniably similar red fruits were only mentioned in an ornamental context.

\section{Different sorting criteria but similar groups}

The phenomenon of similar sorting caused by different criteria has been frequently observed $[9,14,16,42]$. Usually, the coincidence of form and function is explained by a certain form favouring a certain function [9] p. 875, [16] p. 77) or more generally by a "correlation of features that leads them [the informants] to form the same clusters" [14] p. 75. We here put this argument into perspective by using a more comprehensive perception of plant properties. This leads us to the field of sensory perception, which is known to be important for the classification of medicinal plants [46]. Plant perception, which leads to plant classification, happens not only visually, but through all our senses. Auditory perception plays certainly a minor role in the case of plants, but smell, taste and tactile perception are crucial to identify the qualities of a plant. As sight is the dominating human sense especially in western societies, reasoning about categories contains in many cases a strong morphological component. Evaluating plants by taste or smell results likely and directly 
in categories of use (or non-use). The berries, for example, were often put together because of their common use for jam or just because of their edibility. Behind this use-based explanation, a sensory perception argument is hidden: the berries are eaten or used to make jam, because they are all perceived as juicy, sweet and tasty. Other examples are Mentha x piperita and Salvia officinalis, which were rarely separated. They were said to be "kitchen herbs" or "tea herbs", because they are perceived as fragrant species with physiological effects.

Since species of the same plant family tend to share as well morphological as chemical properties, perception with different senses may lead to similar groups. Using the visual and also tactile sense favours morphological explanations, while categories based on taste or smell are likely explained by use.

\section{Conclusions}

Compared with urban populations of western societies, the rural population of the Napf region holds respectable plant knowledge. The folkbotanical classification system of the people is comparable to classification systems of indigenous societies, both in its shallow hierarchical structure and in the amount of recognized taxa.

The classification of plants was mainly guided by morphology, habitat and use. The three aspects may be mutually linked for certain plant groups, which results in always the same groups, independent from the different sorting criteria. Sensory perception allows for a broader explanation of the known coincidence of morphology and use groups. As related plant species share not only morphological, but also chemical properties, perception with different senses (visual, taste, smell) may lead to similar groups.

\section{Consent}

Oral informed consent was obtained from the interviewees or their parents for the publication of this report.

\section{Competing interests}

The authors declare that they have no competing interests.

\section{Author's contribution}

AP and CRV initiated the project and with CSW jointly structured the project and the paper. AP collected and analyzed the data. AP and CSW led the writing. All authors read and approved the final manuscript.

\footnotetext{
Acknowledgements

We would like to thank our interview partners warmly for their time and goodwill, Anna and Maja Monnot for board and lodging and many inside-informations during fieldwork, Franz Huber for redesigning the Anthropac cluster diagram, Susanne Grasser for general exchange of ideas, Elisabeth Danner for the help with the identification of species, Rosmarie and Peter Küchel for the careful affixing of the voucher specimens and three anonymous reviewers for valuable comments and suggestions on additional literature.
}

\section{Author details}

'Department of Sustainable Agricultural Systems, University of Natural Resources and Life Sciences Vienna, (BOKU), Gregor-Mendel-Strasse 33,
A-1180 Vienna, Austria. ${ }^{2}$ Institute of Systematic Botany, University of Zürich, Zollikerstrasse 107, Zürich CH-8008, Switzerland.

Received: 22 May 2014 Accepted: 31 December 2014 Published: 14 March 2015

\section{References}

1. Berlin B. Ethnobiological Classification. New Jersey: Princeton University Press; 1992.

2. Ellen R. The Categorical Impulse: Essays in the Anthropology of Classifying Behaviour. Oxford: Berghahn; 2006.

3. Atran S. Cognitive Foundations of Natural History. London: Cambridge University Press; 1990.

4. Atran S. Folk biology and the anthropology of science: Cognitive universals and cultural particulars. Behav Brain Sci. 1998:21(4):547-609.

5. Ross N, Revilla-Minaya C. Cognitive Studies in Ethnobiology: What can we Learn about the Mind as well as Human Environmental Interaction? In: Anderson EN, Pearsall D, Hunn E, Turner N, editors. Ethnobiology. Hoboken NJ: Wiley-Blackwell; 2011. p. 335-49.

6. Hunn ES, Brown CH. Linguistic Ethnobiology. In: Anderson EN, Pearsall D, Hunn E, Turner N, editors. Ethnobiology. Hoboken NJ: Wiley-Blackwell; 2011. p. 319-33.

7. Holman EW. Domain-specific and general properties of folk classifications. J Ethnobiol. 2005;25(1):71-91.

8. Sanga G, Ortalli G. Nature Knowledge. Ethnoscience, Cognition and Utility. New York and Oxford: Berghahn Books; 2003.

9. Boster JS, Johnson JC. Form or function: A comparison of expert and novice judgements of similarity among fish. Am Anthropol. 1989:91(4):866-89.

10. Agrawal A. Dismantling the divide between indigenous and scientific knowledge. Dev Chang. 1995;26:413-39.

11. Lindemann-Matthies P. Wahrnehmung biologischer Vielfalt im Siedlungsraum durch Schweizer Kinder. In: Klee R, Bayrhuber H, editors. Lehr- und Lernforschung in der Biologiedidaktik. Innsbruck: Studienverlag; 2002. p. $117-30$

12. O'Brien CM. Do they really "know nothing"? An inquiry into ethnobotanica knowledge of students in Arizona, USA. Ethnobotany Res Appl. 2010;8:35-47.

13. Wagner GE. Botanical knowledge of a group of college students in South Carolina. USA Ethnobotany Res Appl. 2008;6:443-58.

14. Medin DL, Lynch EB, Coley JD, Atran S. Categorization and reasoning among tree experts: do all roads lead to Rome? Cogn Psychol. 1997;32:49-96

15. Pardo-de Santayana M, Morales R, Chamomiles in Spain. The Dynamics of Plant Nomenclature. In: Pardo de Santayana M, Pieroni A, Puri RK, editors. Ethnobotany in the New Europe. New York and Oxford: Berghahn Books; 2010. p. 282-306.

16. Nolan JM. Wild plant classification in Little Dixie:Variation in a regional culture. J Ecol Anthropol. 2002;6:69-80.

17. Meilleur BA. Alluetain Ethnoecology and Traditional Economy: The Procurement and Production of Plant Resources in the Northern French Alps. Michigan: UMI Dissertation Information Service; 1986.

18. Atran S, Medin D, Ross N. Evolution and devolution of knowledge: A tale of two biologies. J R Anthropol Inst. 2004;10(2):395-420

19. Yates S, Ramirez-Sosa CR. Ethnobotanical knowledge of Brosimum alicastrum (Moraceae) among urban and rural El Salvadorian adolescents. Econ Bot. 2004;58(1):72-7.

20. Bang M, Medin DL, Atran S. Cultural mosaics and mental models of nature Proc Natl Acad Sci. 2007:104(35):13868-74

21. Nolan JM. The roots of tradition: Social ecology, cultural geography, and medicinal plant knowledge in the Ozark-Ouachita highlands. J Ethnobiol. 1998:18(2):249-69.

22. Federal Office of Meteorology and Climatology MeteoSwiss. www meteoschweiz.ch, Klimanormwerte 1961-1990

23. Delarze R, Gonseth Y. Lebensräume der Schweiz. 2nd ed. Bern: hep Verlag; 2008

24. Welten M, Sutter R. Verbreitungsatlas der Farn- und Blütenpflanzen de Schweiz. Birkhäuser: Basel; 1982

25. Wohlgemuth T. Verbreitungsatlas der Farn- und Blütenpflanzen der Schweiz als elektronische Datenbank. Bot Helv. 1993;103:55-71. http://www.wsl.ch/ land/products/webflora/welcome-de.ehtml.

26. Swiss Federal Statistical Office. http://www.bfs.admin.ch/, Commune portraits data from 2008, national data from 2011. 
27. Weller SC, Romney AK. Systematic Data Collection. Newbury Park CA: Sage Publications; 1988

28. Bernard HR. Research Methods in Anthropology - Qualitative and Quantitative Approches. Walnut Creek: Altamira Press; 2002.

29. Borgatti SP. ANTHROPAC 4.0. Methods Guide. Natick, MA: Analytic Technologies; 1996.

30. Martin GJ. Ethnobotany. A methods manual. London: Chapman \& Hall; 1995.

31. Lauber K, Wagner G, Gygax A. Flora Helvetica. 5th ed. Haupt Verlag: Bern; 2012.

32. Keller F, Lüthi J, Röthlisberger K. Gemüsearten. 2nd ed. Zollikofen: Landwirtschaftliche Lehrmittelzentrale LmZ; 1996.

33. Kellerhals M, Rapillard C, Röthlisberger K, Rusterholz P. Obstsorten. 4th ed. Zollikofen: Landwirtschaftliche Lehrmittelzentrale LmZ; 2003.

34. Neuweiler R, Röthlisberger $K$, Rusterholz P, Terrettaz R. Beeren und besondere Obstarten. Zollikofen: Landwirtschaftliche Lehrmittelzentrale LmZ; 2000.

35. Borgatti SP. ANTHROPAC 4.0. Natick, MA: Analytic Technologies; 1996.

36. Demuth R. Elemente des "Umweltwissens" bei Schülern der Abgangsklassen der Sekundarstufe I. Naturwissenschaften im Unterricht-Chemie. 1992;3:36-8.

37. Cooper CL. Botanical knowledge of a group of South Carolina elementary school students. Ethnobotany Res Appl. 2008;6:123-8.

38. Quinlan M. Considerations for collecting freelists in the field: Examples from ethnobotany. Field Methods. 2005;17:219-34.

39. Zarger RK, Stepp JR. Persistence of botanical knowledge among Tzeltal Maya children. Curr Anthropol. 2004:45:413-8.

40. Brun-Hool J. Vom Alisli zum Zyterööseli. Mundartnamen der Pflanzen im Amt Willisau. Heimatkunde des Wiggertals. 2004;62:11-29.

41. Brun-Hool J. Bauerngärten im Kanton Luzern. Mitteilungen der Naturforschenden Gesellschaft Luzern. 1984;28:3-191.

42. Medin DL, Ross N, Atran S, Cox D, Coley J, Proffitt JB, et al. Folkbiology of Freshwater Fish. Cognition. 2006;99(3):237-73.

43. Ellen R. Ethnomycology among the nuaulu of the Moluccas: putting Berlin's "general principles" of ethnobiological classification to the test. Econ Bot. 2008;62(3):483-96.

44. Tunnicliffe SD, Reiss MJ. Building a model of the environment: how do children see plants? J Biol Educ. 2000;34(4):172-7.

45. Grasser S, Schunko C, Vogl CR. Gathering "tea" - from necessity to connectedness with nature. Local knowledge about wild plant gathering in the Biosphere Reserve Grosses Walsertal (Austria). J Ethnobiol Ethnomedicine. 2012;8:31.

46. Shepard $\mathrm{jr} \mathrm{GH}$. A sensory ecology of medicinal plant therapy in two amazonian societies. Am Anthropol. 2004;106(2):252-66.

doi:10.1186/1746-4269-11-13

Cite this article as: Poncet et al:: Folkbotanical classification: morphological, ecological and utilitarian characterization of plants in the Napf region, Switzerland. Journal of Ethnobiology and Ethnomedicine 2015 11:13

\section{Submit your next manuscript to BioMed Central and take full advantage of:}

- Convenient online submission

- Thorough peer review

- No space constraints or color figure charges

- Immediate publication on acceptance

- Inclusion in PubMed, CAS, Scopus and Google Scholar

- Research which is freely available for redistribution 